The following alphabetical and geographical lists represent NALLD membership through February 4, 1983 . Please send corrections directly to:

\author{
NALLD \\ Academic Publications \\ Administration Building \\ University of Louisvilile \\ Louisville, KY 40292 USA
}

This copyrighted membership list is the property of the International Association for Learning Laboratories (IALL) and may not be used for commercial purposes without specific permission of IALL. For information concerning the use of this list, contact the Editor of the NALLD Journal:

\author{
Dale V. Lally, Jr. Director \\ Arts and Sciences Learning Laboratory \\ 16 Bingham Humanities Building \\ University of Louisville \\ Louisville, KY 40292 USA
}

ALPHABETICAL LIST

ADFL Bulletin, Editor, 62 Fifth Avenue, New York, NY, 10011

AV Language Journal, Attn: A. P. Dyson, University of Oxford, Lang. Ctr., 41 Wellington Square, Oxford, ENGLAND OX1 2JF

Abed, A. Z., Foreign Language Laboratory, Louisiana State University, Baton Rouge, LA, 70803

Abraham, Ruth, Director, Language Lab., Bowdoin College, Sills Hall, Brunswick, ME, 04011

Abrams, W., Education Department, Capital Complex, Carson City, NV, 89710

Achiba, Machiko, 8-34 Komuinshukusha, 11-72 Sakashita, Myodaiji-cho, Okazaki, Aichi, JAPAN 444

Aikens, H. F., Part-time Studies \& Extension, Dalhousie University, Halifax, NS, CANADA B3H $3 J 5$

Al aghelley, Abdulaziz M., 200 N. Negley Ave., Apt. E-42, Pittsburgh, PA, 15206

Al len, Andrew, Romance Languages Dept., University of Tennessee, Knoxville, TN, 37996

Al len, Ernest, Language Laboratory, Texas Christian University, 1 Allen Farm Road, Fort Worth, TX, 76116

Allen, Nina A., Language Learning Center, $\mathrm{OH}-40$, University of Washington, Seattle, WA, 98195

Altman, Howard B., Dept. of Modern Languages, University of Louisville, Louisville, KY, 40292

Alyta, Kenneth J., 5316 Chedworth Orive, Charlotte, NC, 28210

Amano, Kazuo, 16--6 Shiomigaoka-Cho, Chiba-Shi 280, JAPAN

Ambard, Francois, Colorado Apts., K201, Lake Aust in Blvd., Austin, TX, 78703

Annamalai, E., I/C Library, Central Inst. of Indian Languages, Manasagangotri, Mysore-570 006, INDIA

Appalachian State University, Serials Department, Bulk Library, Boone, NC, 28608

Applied Ling. Institute of Belgium, Attn: Willy Martin, Blijde Inkomststraat 21, Leuven, BELGIUM 3000 
Arizona State University, Library - Periodicals, Tempe, AZ, 85281

Arnold, E. P., 201 Strode Tower, Clemson University, Ciemson, SC, 29631

Arnold, Robert L., Instructional Resources, University of Central Florida, Box 25,000, Orlando, FL, 32816

Aronovsky, Mrs., Bishop Hartley High School, 1285 Zettler Road, Columbus, $\mathrm{OH}, 43227$

Assoc. for Educational Comm., Attention: John J. Faber, 1201 Sixteenth St., N.W., Washington, DC, 20036

Aubert, Jean-Philippe, McGill University, 3438, McTavish Street, Montreal, CANADA H3A IX9

Auchmuty Library, Serials Dept., University of Newcastle, Newcastle, NSW, AUSTRALIA 2308

Aucoin, G. E., 34 Langley Avenue, Dartmouth, Nova Scotia, CANADA B2W 2 Y6

Augerot, James E., Language Learning Center, University of Washington, Seattle, WA, 98195

Aulestia, Victor H., Language Media Center, University of Maryland, 5401 Wilkens Avenue, Baltimore, MD, 21228

Bailey, Leslie F., Language Laboratories, 48B Kresge, 1859 Sheridan Rd., Northwestern University, Evanston, IL, 60201

Baker, Reid E., Foreign Language Supervisor, Education Department, 65 South Front Street, Columbus, $\mathrm{OH}, 43215$

Balcaen, Hubert L., Language Laboratories, University of Manitoba, Winnipeg, CANADA R3T 2 M8

Baltra, Liliana, (CPEIP), Casilla 16162, Santiago, CHILE 9

Barakat, Ghias, P.0. Box 8333, Austin, TX, 78712

Barnes, J., Department of Education, Agana, GU, 96910

Barria, Jose G., 4521 Leeds Avenue, El Paso, TX, 79903

Bartz, W., Public Instruction, State House 229, Indianapolis, IN, 46204

Bator, Joseph W., Language Development, Pan American World Airways John F. Kennedy Airport, Jamaica, NY, 11430

Bell, Anne B., 4903 44th Avenue, Moline, IL, 61265

Bennet, T.J.A., Language Laboratories, Hochschule St. Gallen, Dufourstrasse 50, St. Gallen, SWITZERLAND 9014

Benseler, David P., MODERN LANGUAGE JOURNAL, 314 Cunz Hall, Ohio State University, Columbus, $\mathrm{OH}, \mathbf{4 3 2 1 0}$

Berndt, Robert J., Dept. of Foreign Language, Southwest Missouri State University, Springfield, MO, 65802

Bernier, Marc, 2164 Florida Avenue, NW, Washington, DC, 20008

Beusch, A., Department of Education, P.0. Box 8717, BWI Airport, Baitimore, MD, 21240

Beveridge, Martha J., 8127 Birch Street, New Orleans, LA, 70118

Bleznick, Donald W., Editor, HISPANIA, Romance Languages, University of Cincinnati, Cincinnati, $\mathrm{OH}, 45221$

Blinco, Priscilla N., 196 Stewart Drive, Tíburon, CA, 94920

Bloomer, Joan, Coordinator, Langauage Lab, University of California Davis, Davis, CA, 95616

Bocaz-Moraga, Sergio, Dept. of Modern Languages, Eastern Washington University, 3144 Patterson Ha11, Cheney, WA, 99004

Boggins, Judy, Foreign Language Lab, Raymond Waiters College, 9555 Plainfield Road, Cincinnati, $\mathrm{OH}, 45221$

Booth, Trudie, Dept. Of French, University of Portland, $5000 \mathrm{~N}$. Willamete Bivd., Portland, OR, 97203

Bosworth, Lewis A., New Student Services, 905 Univ. Ave., Suite 1, University of Wisconsin, Madison, WI, 53715

Boudreau, Jacqueline, Language Laboratory, Wheaton College, Norton, MA, 02766

Bowen, Louise H., 201 Bart lett Ha 11, Dartmouth College, Hanover, NH, 03755

Bowie State College, Thurgood Marshall Library, Pullen Library Periodicals, Bowie, MD, 20715 
Boyden, Patrick C., Self-Instruction Center, 271 Library, Kent State University, Kent, $\mathrm{OH}, 44242$

Brandon University, John E. Robbins Library, Brandon Man., CANADA R7A6A9

Bridgers, William K., Learning Laboratories, Instructional Development, University of California, Santa Barbara, CA, 93106

Brigham Young University, Dr. Jerry W. Larson, Humanities Research Center, 1308-34, Provo, UT, 84602

Brigham Young University, Harold Lee Library, Serials Section, Provo, UT, 84602

Brinton, Donna, ESL Media Consultant-UCLA, English IESL, 405 Hilgard Avenue, Los Angeles, CA, 90024

British Library, Lending Division, Accessions Department-Lending Div., Boston Spa, Wetherby, Yorkshire, ENGLAND LS237BQ

Bronx Community College-CUNY, Dept. Library \& Learning Resources, University Ave. \& W 181 St., Bronx, NY, 10453

Brown, C. P., Foreign Language Department, Ivan Wilson Building 251, Western Kentucky University, Bowling Green, KY, 42101

Brown University, Language Laboratory Library, Box E, Providence, RI, 02912

Buchhandlung Lehmkuhl, Leopoldstrasse 45, 8000 Muchen 40, WEST GERMANY

Burggraaf, Samuel E., W- 162 Stadium, Brigham Young University, Provo, UT, 84602

Butler, Samuel R., 1095 South Downing Street, Denver, C0, 80209

Caldwell, William A., The American University, LFS Asbury, Rm. 326, 4400 Massachusetts Ave., NW, Washington, DC, 20016

California Polytechnic State Univ., University Library - Periodicals, San Luis Obispo, CA, 93407

Calvin College Library, 3207 Burton, S. E., Grand Rapids, MI, 49506

Canadian Forces Base St. - Jean, Base Commander, Unit B - Rm. F-267, Richelain, Quebec JOJ IRO CANADA

Capretz, Pierre J., Director, Language Lab., Yale University, 111 Grove Street, New Haven, CT, 06511

Carleton College, Library, Northf ield, MN, 55057

Carlson, Florence, Director, Language Lab., Wellesley College, Wellesley, MA, 02181

Caron, Paul, Dept. of English, CEGEP Riviere du Loup, 80 Frontenac, Riviere du Loup, Quebec 65R IV8 CANADA

Carter, Thomas P., Learning Resource Services, Dalhousie University, Killam Library, Hal ifax, NS, CANADA B3H 4 H8

Cate, Robert L., Modern Language Laboratory, University of Texas at El Paso, El Paso, TX, 79968

Center for Information on, Language Teaching, Deputy Librarian, 20 Carlton House Terrace, London S.W., ENGLAND IY 5AP

Chang, Gerald K. J., Foreign Lanaguage Laboratory, Moore Hall 256, 1890 East-West Road, Honoluiu, HI, 96822

Cheatham, Rosalie M., Language Laboratory, Arkansas University at Little Rock, 6405 Evergreen Road, Little Rock, AR, 72207

Cherry, Charles Maurice, Modern Language Laboratory, Fuman University, Greenville, SC, 29613

Chestnut, D., Education Department, Education Building, Box 911, Harrisburg, PA, 17126

Christman, Sandra, Foreign Language Department, Chillicothe High School, 381 Yoctangee Pkwy., Cillicothe, OH, 45601

Ciceran, A. J., Language Laboratory, Brock University - Decew Campus, St. Catharines, Ontario, CANADA L2S $3 A 1$

$\mathrm{Cl}$ ark, C. A., Australian National University, Instructional Resources Unit, Chifley Library, Box 4, Canberra, A C T, AUSTRALIA 2600

Clark, D. J., Gilman Hal1, Johns Hopk ins University, Baltimore, MD, 21218 
Cleveland State University, Library Order Unit, Serials, 1860 E. 22nd Street, Cleveland, $\mathrm{OH}, 44115$

Coffey, Thomas F., Classics \& Modern Languages Dept., Creighton University, Onaha, NE, 68178

College of Cape Breton, Library - Sydney Campus, Box 5300, Sydney, NS, CANADA BIP 6L2

Coltharp, Jerry, Audio Visual Service, 340 Gaskill Hall, Miami University, Oxford, $\mathrm{OH}, 45056$

Columbus Memorial Library, Serials Pub Sec, OAS, 17th \& Constitution, Washington, DC, 20006

Comet, Honore, All-World Language Institute, Language Laboratory - Suite 500, 280 South Beverly Drive, Beverly Hills, CA, 90212

Concordia University, Libraries-Serials-Acquisitions, P.0. Box 2650, Montreal, Que., CANADA H3G 2P7

Conner, Maurice W., Central States Conference, University of Nebraska, P. 0 . Box 688, Omaha, NE, 68101

Conwell, Marilyn J., 251 West DeKalb Pike, Apt. 909C, King of Prussia, $P A, 19406$

Couroux, Gerard, College Marie Victorin, 7000 rue Marie Victorin, Montreal, Que., CANADA 462

Courtright Memorial Library, Otterbein College SAN 313-7937, Westerville, $\mathrm{OH}, 43081$

Cox, Shirley 0., Language Laboratory, Auburn University, Auburn, AL, 36830

Creamer, Jr., John F., Director, Language Lab, Hudson High School, Brigham Street, Hudson, ME, 01749

Criminale, Leonard R., Language Laboratory, Elmira College, Elmira, NY, 14901

Crosbie, K., Bilingual Instruction, Public Instruction, 01d Capitol Building, 0lympia, WA, 98504

D.H. Hill Library, Periodicals Service Center, Box 5007-NC State, Raleigh, NC, 27650

OLIWC ACademic Library, DLIFLC B1dg. 618, M/F DAKF03-80-M-3315, Presidio, Monterey, CA, 93940

Daigaku, Gifu Jyoshi Tanki, 2693 Fukumitsu, Nagara, Gifu 502 MZ, JAPAN

Daigaku, Kyushu Sangyo, (LL Room), 327, Matugadai-2-chome, Higashi-ku, Fukuoka City, JAPAN 813 (MZ)

Daistrom, E. Kay, University of Nebraska, Foreign Languages, Omaha, NE, 68112

Dammer, P., Foreign Language Education, Education Department, Albany, NY, 12234

David Yellin Teacher's Seminary, Beth-Hakerem, P.0. Box 3578, Jerusalem, ISRAEL

Davies, Norman F., Professor, University of Linkoping, Linköping, SWEDEN S-581 83

de Buhler, Angela Vidal, Pampa 1940, 1428 Buenos Aires, ARGENTINA

de Johnson, Teresa H., Department of Modern Languages, St. Louis University, St. Louis, M0, 63103

de la Portilla, Marta, Spanish Department, Manhattanville College, Purchase Street, Purchase, NY, 10577

DeMers, Irene, Harvard School, 3700 Coldwater Canyon Road, North Hollywood, CA, 91604

DeTorre, Emilio E., Queens College, City University of New York (CUNY), Flushing, NY, 11367

Dente, Edmund, Conte Language Lab, Tufts University, Cabot Center, Rm. 202, Medford, MA, 02155

Department of Education, Director, Bilingual Education, Boston, MA, 02111

Di11, David, Foreign Language Dept., Lancaster High School, 1312 Granville Pike, Lancaster, $\mathrm{OH}, 43130$ 
Dinkelacker, Horst, Language Center, Southwestern at Memphis, $2000 \mathrm{~N}$. Parkway, Memphis, TN, 38112

Dodge, James W., Secretary/Treas., Northeast Conf., Box 623, Middlebury, VT, 05753

Dowling, J. K., Language Laboratory, University of Queensland, St. Lucia, Bris, AUSTRALIA 4067

DuVerlie, Claude, Modern Languages and Liguistics, UMBC, Catonsville, MD, 21228

ELS Educational Media, Attn: Shelby Hardage, P. 0. Box 20604, Ok lahoma City, OK, 73156

ERIC Exchange, Center for Applied Linguistics, 3520 Prospect Street, N.W., Washington, DC, 20007

Earle, Betty B., Language Lab, Newton South High School, Needham, MA, 02192

Education Language Dept., 1067 Buford Ellington, Kennedy Campus, Memphis, TN, 38111

Educational Electronics of Calif., Attn: Bernard Keach, 213 North Cedar Avenue, Inglewood, CA, 90301

Educational Media Division, Attn: Jim Goodin, P. 0. Box 20604, 2812 Quail Plaza Drive, Ok lahoma City, OK, 73156

Elmendorf, William E., Language Laboratory, Western Washington State College, Bellingham, WA, 98225

Enos, Jacqueline, Foreign Language Dept., Bridgewater State College, Bridgewater, MA, 02324

Entin, Nathaniel A., Gratz College, 10th Street \& Tabor Road, Philadelphia, PA, 19141

Feagin, C., Department of Education, Education Annex-Trinity, At lanta, GA, 30303

Fisher, Ronald D., Language Laboratory, LaSalle College, 20th Street at Olney Avenue, Philadelphia, PA, 19141

Florida State University, R. M. Strozier Library, Serials Department, Tallahassee, FL, 32306

Ford, Kathleen, Administrative Assistant, Foreign Language - Instruc. Lab., 362 Royce Hall, UCLA, Los Angeles, CA, 90024

Forth, Joli, 973 Kingston Avenue, Piedmont, CA, 94611

Fournier, R., Bilingual Education, 64 N. Main Street, 3rd Floor, Concord, $\mathrm{NH}, 03301$

Fox, Jean, Language Department, Columbus School for Girls, 56 South Columbia Avenue, Columbus, $\mathrm{OH}, 43209$

Francis, Larry P., Intensive English Program, University of Missouri, 228 Gentry, Columbia, MO, 65211

Francis H. Green Library, West Chester State College, West Chester, PA, 19380

Frank, Stefania, University Hall, Bowling Green State University, Bowling Green, $\mathrm{OH}, 43402$

Frechette, Ernest A., Foreign Language Education, Florida State University, Tallahassee, FL, 32306

Free University of Berlin, Foreign Language Laboratories, Habelschwerdter Al lee 45, Innenstrasse 24, 1 Berl in 33, WEST GERMANY

Freudenstein, Reinhold, Foreign Language Research Info. Ctr., Lahnberge, D-3550 Marburg, WEST GERMANY

Fritsch, Hans J., P.0. Box 61, Whately, MA, 01093

Froehlich, Jurgen, Language Laboratory, Pomona College, Claremont, CA, 91711

Frost, Arthur F., Director, Language Lab., Princeton University, 304 E. Pyne Building, Princeton, NJ, 08540

Fukuoka University, LL Room, Nanakuma, Fukuoka 814 MZ, JAPAN 
Galloway, V., Foreign Language Consultant, Education Department, 801 Rutledge, Columbia, SC, 29201

Garinger, Ermal E., Language Laboratories, University of Kansas, 4069 Wescoe Hall, Lawrence, KS, 66045

Gennaula, M. G., 5872 Kings School Road, Bethel Park, PA, 15102

Gilde, Hans M., Oldfather 1111 , University of Nebraska, Lincoln, NE, 68588

Gillespie, Netta, Language Laboratory, University of II linois, G-89 Foreign Languages, Urbana, IL, 61801

Gilmore, Roger H., As soc. Prof. of Spanish, Colorado State University/, Foreign Languages, Fort Collins, C0, 80523

Gionet, Arthur J., Dept. of Foreign Languages \& Lit., North Texas State University, Denton, TX, 76203

Gleaves, Glen, Language Laboratory, University of South Alabama, Mobile, $A L, 36688$

Goldsworth, Thomas, P.0. Box 537, Odessa, FL, 33556

Gore, Peter B., P.0. Box 22, Wilmington, VT, 05363

Goulet, Sister Noella, Notre Dame College, 2321 Elm Street, Manchester, $\mathrm{NH}, 03104$

Governors State University, University Library, Serials Dept. - Kardex, Park Forest S., IL, 60466

Grant, R. M., 2345 Filbert Street, No. 202, San Francisco, CA, 94123

Griffin, Howard, Denison University-A/V Services, Granvili, OH, 43023

Griffith, P., Education, 100 North First Street, Springfield, IL, 62777

Grittner, F., Foreign Language Education, Public Instruction, 126 Langdon, Madison, WI, 53702

Hall, Bunelle M., Monterey Institute of International Studies, 425 Van Buren Street, Monterey, CA, 93940

Hamilton College, Serials Dept., Clinton, NY, 13323

Hamm, C., Language Laboratories, Queen's University, Kingston, Ont., CANADA K7L 3N6

Hamerly, Hector, Simon Fraser University, Literature \& Linguistics, Burnaby, BC, CANADA V5A IS6

Hanby, Mr., Principal, New Lexington Jr. High School, Route 2, New Lexington, $\mathrm{OH}, 43764$

Hanzel, Louis F., Nanzan University, 18 Yamazato-cho, Showa-ku, Nagoya, JAPAN 466

Harnagle, James, Language Department, University of Steubenville, College Heights, Steubenville, $\mathrm{OH}, 43952$

Harwood, Sharon, Dept. of Foreign Language, Memphis State University, Memphis, TN, 38152

Heather, Anthony J., Language Resource Center, Loyola University, Chicago, IL, 60626

Heilenman, Laura K., Dept. of French \& Italian, Northwestern University, Evanston, IL, 60201

Heinle \& Heinle Publishers, Inc., Attn: Charles Heinle, 286 Congress Street, Boston, MA, 02210

Helsingin Yliopisto, Kielikeskus, Fabianinkatu 26, SF-00100, Helsinki 10, FINLAND

Hendriks, Helga M. Leonhardt, Foreign Language Insturctor, 640 San Elijo Street, San Diego, CA, 92106

Hennig, C. W., Language Laboratory, University of Waterloo, Waterloo, Ont., CANADA N2L 3GI

Herdler, Mrs., Romance Language Dept., Walnut Hills High School, 3250 Victory Pkwy., Cincinnati, OH, 45207

Herrera, Lazaro M., Language Laboratory, The Westminster Schools, $1424 \mathrm{~W}$. Paces Ferry Road, N.W., At lanta, GA, 30327

Hershel, Kenneth, 3222 Murray Hill Pike, Louisville, KY 40222

Hewson, J., Linguistics, Memorial University of Newfoundland, St. John's NFD, CANADA 
Hill, Ann R., Language Laboratory, Randolph-Macon Woman's College, 2400 Rivermont Avenue, Lynchburg, VA, 24503

Hiroshima Daigaku, Honbu Toshokan-K, Higashisendamachi Nakaku, Hiroshima MZ, JAPAN 730

Hiroshima Shudo Daigaku Library, Otsuka Numata-Cho, Hiroshima (Kin), JAPAN 731-31

Hirsch, Bernice G., Foreign Language Lab, Samford University, 800 Lakeshore Drive, Birmingham, AL, 35229

Hockenson, Mrs., Language Lab, Cincinnati Country Day School, 6905 Given Road, Cincinnati, OH, 45243

Hoff, Roma, University of Wisconsin-Eau Claire, 335 Bartlett Court, Eau Claire, WI, 54701

Holmes, Glyn, French, University of Western Ontario, London, CANADA N6A $3 K 7$

Honda, Ritsuri, Assumption College, 2-16 Ote-cho, Takatsuki-shi, Osaka, JAPAN 569

Howard, J., Department of Education, 111 Coliseum Blvd., Montgomery, AL, 36109

Howard University, Language Lab/903301, Room 361 Locke Hall, 2441 6th Street, N.W., Washington, DC, 20059

Howard University Library, Acquisitions Dept. Serials, Room \#105, Washington, DC, 20059

Howe, E., Foreign Language Education, Education Department, 250 East 500 South, Salt Lake City, UT, 84111

Hudson, G., Department of Education, Little Rock, AR, 72201

Hughes, Ann M., Language Lab, Loyola College in Maryland, 4501 N. Charles St., Baltimore, MD, 21210

Hughett, Harvey, Dept. of Foreign Languages \& Lit., College of Letters \& Science, University of Idaho, Moscow, ID, 83843

Hutchinson, Joseph C., P.0. Box OJ, Pacific Grove, CA, 93950

Hyogo-Kyoiku Daigaku, Toshokan, Yashiro-Cho Yashiro, Kato-Gun Hyogo, JAPAN 673-14

Ibaraki Christ-Kyo, Tanki-Daigaku Library, 6-11-1 Omika-Cho Hitachi, Ibarak i 319-12, JAPAN (KIN)

Ikeura, Sadahiko, Fukuoka University of Education, 279 Akama, Maunakata-gun, Fukuoka, JAPAN

Infante, J., Languages, Public Instruction Department, Raleigh, NC, 27611

Institue de Linguistique Appliquee, Laboratoires de Langues, Universite de Strasbourg, 22, rue Descartes, Strasbourg-Cedx, FRANCE 67084

Instructional Materiais Center, 201 East Nine Mile Road, Highland Spring, $V A, 23075$

Inter-American University, Library, San German, PR, 00753

Iowa State University, English/IEOP, 203 Ross Hall, Anes, IA, 50011

Irving, Evelyn Uhrhan, 2508 Glen Elm Drive, N. E., Cedar Rapids, IA, 52402

Ishikawa Kosen, Kitachujo, Tsubata, Kahoku-Gun, Ishikawa, JAPAN 929-03 MZ

Jabalquinto, Sergio Rios, Casilia 165, Tarija, B0LIVIA

Jackson, D., Second Languages, Public Instruction, Helena, MT, 59601

Jackson, Gordon, 257 Cliffview Drive, Gahanna, OH, 43230

Jebe, S., Department of Education, 649 Capitol Square, 550 Cedar Street, St. Paul, MN, 55101

Jewish National \& Univ. Lib., Attn: Periodicals Dept., P.0. Box 503, Jerusalem, ISRAEL 91004

Johansen, Kjell M., Language Laboratory, FL Dept., North Texas State University, Denton, TX, 76203

Johnson, Barbara, Foreign Lanaguage Department, Washington State University, Pullman, WA, 99164

Johnson, Tom, Audio-Visual Marketing, TELEX COMMUNICATIONS, INC., 9600 Aldrich Avenue South, Minneapolis, MN, 55420 
Jo int National Comn. for Languages, J. David Edwards, Director, 11 DuPont Circle, N.W., Suite 210, Washington, DC, 20036

Jordan, Terence C., Language Centre, Griffith University, Nathan, Queens., AUSTRÁLIA 4111

Kagawa University, Library, Saiwai-Cho, Takamatsu-Shi, JAPAN 760 (KIN)

Kalenchic, Mary Beth, Learning Center, Lehigh University, 401 Coppee Hall 33, Bethlehem, $P A, 18015$

Kamehameha Schools, Attn: Bill Gemmer, Kapalma Heights, Honolulu, HI, 96817

Kansai University Library, P.0. Box 50, Suita, 0saka, JAPAN 564 MZ

Kenagawa University, Library, Rokkakubashi, Kanagawa-Ku, Yokohama (MZ), JAPAN 221

Kenji Kitao, Dept. of English, Doshisha University, Kyoto, JAPAN 602

Kenner, R., $\mathrm{H}-399$ Concordia University, Language Laboratory Division-AV, 1455 de Maisonneuve Blvd., Montreai, Que., CANADA H3G IM8

Kessler, Michael, School of Humanities, 1600 Holloway, San Francisco State University, San Francisco, CA, 94132

King, R., Education Department, P. O. Box 480, Jefferson City, M0, 65102

Kipphorn, Jr., Richard A., Language Laboratory, Saint Joseph's University, Philadelphia, PA, 19131

Kirk, David, 124 Chace Street, Santa Cruz, CA, 95060

Knox, H. Todd, Language Laboratory, University of Southwestern Louisiana, Box 43331, Laf ayette, LA, 70506

Koester, A., Department of Education, Frankfort, KY, 40601

Kogut, Daniel E., Wickersham Hall, Millersville State College, Millersville, PA, 17551

Kohmoto, Sutesaburo, 4-16-17 Meguro, Meguro-ku, Tokyo, JAPAN 153

Konyvtar, Egyetemi, POB 483, Budapest 1372, HUNGARY

Korkeakoulun, Joensuun, Kirjasto, PL 111, 80101, Joensuu 10, FINLAND

Kozoriz, G., Canadian Forces Lang. Sch 1-0ttowa, Nat'1. Defense Hdq. (NDHQ), Ottowa, Ontario, CANADA KIA OK2

Krupatkin, Yakov Borisovich, U.1. Odesskaja 1, kv. 19, Sevastopol, USSR 335000

Kruse, Jr., E. L., 733 Kirkwood Drive, Grand Is land, NY, 14072

Kump, Richard J., 1822 Ricardo Drive, Cape Girardeau, M0, 63701

Kure Koygo Koto, Senmon Gakko Library, Aga-Minami-2-2-11, Kure, JAPAN 737 MZ

Kuroda, Takashi, Language Laboratory Assoc. of Japan, Eng. Lit. Dept. Otsuma Womens Col., 12, 3-bancho, Chiyoda-ku, Tokyo, JAPAN 102

Kurtz, Don, Language Learning Center, Department of Foreign Languages, Box 3L, Las Cruces, NM, 88003

Kyoto Sangyo Daigaku, Toshokan, (Kyoyo-Eigo-Ken), Kita-Ku Kyoto, JAPAN $603 \mathrm{MZ}$

LHTEC, 905 Lindsey Hopkins Library, 1410 NE 2nd Avenue, Miami, FL, 33132

Lavallee-Wms., Marthe, 1326 Spruce Street, No. 3001, Philadelphia, PA, 19107

Labs for Recorded Instruction, Attn: Dr. R. G. Gilgen, 279 Van Hise Hall, 1220 Linden Drive, Univ. of Wisc., Madison, WI, 53706

Lacher, S., Languages, Public Instruction, Capitol Building, Bismarck, ND, 58505

Lally, Jr., D. V., Arts \& Sciences Learning Lab, University of Louisville, Louisville, KY, 40292

Lance, John, Japanese American Conversation Inst, Intl. Education Center, 21 Yotsuya, 1-chome, Shinjuku-ku, JAPAN 160

Lange, Dale L., University of Minnesota, 130B Peik Hall, 159 Pillsbury Dr., S.E., Minneapolis, MN, 55455

Language \& Language Behavior Abs., P.0. Box 22206, San Diego, CA, 92122 
Language Instruction Supervisor, Bilingual Education, P.0. Box 2219, Hartford, CT, 06115

Language Instruction Supervisor, Education Department, P.0. Box 630, St. Thomas, VI, 00801

Laroche, J., Languages, D.C. Public Schools, 415 12th Street, N.W., Washington, DC, 20004

Larson, H. M., Languages \& Literatures, Cedarville College, Cedarville, $\mathrm{OH}, 45374$

Latinovich, Gina, Language Lab, Marquette University, 526 N. Fourteenth St., Milwaukee, WI, 53233

Lawrason, Robin E., Media Learning Center, Temple University, Philadelphia, PA, 19122

Lemyze, J. C., Language Laboratory, McGill University, Peterson Hall, 3460 McTavish Street, Montreal, Que., CANADA H3A IX9

Levinson, Bernice, Language Laboratory, Brooklyn College-CUNY, Modern Languages \& Literatures, Brooklyn, NY, 11210

Lianez, David, Language Lab, Van Wert High School, Crawford \& Jefferson, Van Wert, $\mathrm{OH}, 45891$

Library of Congress, Order Division, Attn: Section C, Washington, DC, 20540

Lindsey Hopkins, Library 82, L.H.T.E.C., 750 N.W. 20th Street, Miami, FL, 33136

Little, William A., GERMAN QUARTERLY, Germanic and Slavic Languages, University of Virginia, Charlottesville, VA, 22901

Lopez, Jose A., Licenciado, ESPOL, Apartado 5863, Guayaquil, ECUADOR 303733

Loud, Mary, Foreign Languages, Youngstown State University, Youngstown, $\mathrm{OH}, 44555$

Luckau, Paul F., Germanic Languages, Brigham Young University, 270 MSRD, Provo, UT, 84602

Macinko, John, Language Laboratories, University of Colorado, Hellems 156, Box 239, Boulder, CO, 80309

Magi 11, Robert, Language Lab, Dept. of Modern Language, Towson State University, Baltimore, MD, 21204

Majhanovich, Suzanne E., Faculty of Education, University of Windsor, Windsor, Ont., CANADA N9B $3 P 4$

Marietta Coillege, Modern Language Dept., Marietta, OH, 45760

Marino, Anna, Language Laboratory, Hunter College, 695 Park Avenue, Box 375, New York, NY, 10021

Martin, Leslie, Columbia-Green Community College, P.0. Box 1000, Hudson, NY, 12534

Marxheimer, Edward, Romance Languages, University of Alberta, Edmonton, Alberta, CANADA T6G 2E6

Mason, Richard G., 403 N. Franklin Street, P. 0. Box 26, Houghton, MI, 49931

Maynes, J., Department of Education, 1535 West Jefferson, Phoenix, AZ, 85007

Maze, Louis M., English Language Program Director, Procter \& Gamble Sunhome Mfg. Co., 321 Yawata-machi, Takasaki-shi, JAPAN 370

McAndrew, Alex, Univ. of Sydney-Language Study Ctr., Sydney, NSW, AUSTRALIA 2006

McCarty, Jean, Language Laboratory, 106 Calkins Hall, Hofstra University, Hempstead, NY, 11550

McCune, Jerry, Language Laboratory, Indiana University, Ballantine Hall 120, Bloomington, IN, 47401

McGill University Libraries, Serials Department, 3459 McTavish Street, Montreal, CANADA H3A IYi

Mckay, Jym M., Dept. of Speech Communication, University of Texas, Austin, $T \dot{X}, 78712$ 
McNeese State University, Frazar Memorial Library, Serials Department, Lake Charles, LA, 70609

Mellgren, Millie, 3009 F Street, Lincoln, NE 68510

Meneghini, Marc, 7214 St. Charles Avenue, New Orleans, LA, 70118

Menin, J. L., Director, Language Laboratory, Rice University, P.0. Box 1892, Houston, TX, 77251

Mercer University, Language Lab, Attn: Chairman, Dept. of Modern Foreign Languages, Macon, GA, 31207

Messner, C. A., Modern Language, Carleton College, Northfield, MN, 55057

Metcalfe, John, Director, Stanford University Language Lab, Stanford, CA, 94305

Miller, J. B., Wittenberg University, Springfield, OH, 45501

Miller, William I., Modern Languages, University of Akron, 31001 in Hall, Akron, $\mathrm{OH}, 44325$

Ministry of Culture \& Recreation, Resource Centre, 77 Bloor Street West, Toronto, Ontar., CANADA M7A 2R9

Modern Language Association, Advertising Manager, 62 Fifth Avenue, New York, NY, 10011

Modlin, Columbus M., 2817 W. North Ave., Baltimore, MD, 21216

Monash University, Periodicals Department, Clayton, Vic, AUSTRALIA 3168

Moorhead State University, Library Serials Department, Moorhead, MN, 56560

Morris Brown College, Plant Operations-Receiving, 643 Martin Luther King, Jr., Dr., At lanta, GA, 30314

Morrison., Robert R., Southern Missionary College, P.0. Box 475, Collegedale, TN, 37315

Moseby, Erik, Tandberg A/S, P. 0. Box 53, 2007-KJELLER, NORWAY

Mostek, Karlene, 1625 W. Lunt, Chicago, IL, 60626

Murray, David R., AV Services, University of Kentucky, 111 Scott St. Bldg., Lexington, KY, 40506

Nagoya Gakuin Daigaku, Gaikokugo Center, Kamishinano-Cho, Seto, Aichiken 48012MZ, JAPAN

Nassau Cormunity College, Library - Periodicals Unit, Stewart Avenue, Garden City, NY, 11530

National Institute of Education, Educational Research Library, Serials Section, Washington, DC, 20208

National Library of Australia, Preliminary Processing, (NS 110/72), ACT 2600, Canberra, AUSTRALIA

National Library of Canada, Order Section, 395 Wellington Street, Ottawa, Ontario, CANADA KIA ON4

National University of Singapore, Central Library, Serials Department, Kent Ridge, SINGAPORE

Nelson, R., College Department, John Wiley \& Sons, 605 3rd Avenue, New York, NY, 10016

Nicholson, C., Department of Education, 120 East 10th Street, Topeka, KS, 66612

Nicholson, Cynthia, Supervisor, Language Lab., Boston College, Lyons 313, Chestnut Hil1, MA, 02167

Nielson, M., Education Department, 301 Centennial Mall South, Lincoln, NE, 68509

Nihon Eigo-Kentei-Kyokai, I Yaraicho, Shinjuku-ku, Tokyo, JAPAN 162 (SSD)

Norrish, N., Language Laboratory, University of Victoria, Private Bag, Welington, NEW ZEALAND

North Texas State University, Library, P. O. Box 5188 NT Station, Denton, $T X, 76203$

Northeastern University, Learning Resources Center, 406 Dodge, 360 Huntington Ave., Boston, MA, 02115

Norton, Jeffrey, Jeffrey Norton Publishers, Inc., Audio-Forum, 145 E. 49th Street, New York, NY, 10017 
Novak, Sigrid Scholtz, Dept. of Modern Languages, McNeese State University, Lake Charles, LA, 70609

Nuffer, J. Stanley, Learning Laboratory, Portland State University, P.0. Box 751, Portland, OR, 97207

O'Brien, Paula Sullivan, Dept. of Linguistics/ESL, Trinity College, 124 Michigan Ave., NE, Washington, OC, 20017

O'Donohue, Barbara, Communications, Hiroshima Bunkyo Womens College, Kabe Cho-Asa Kita Ku, Hiroshima, JAPAN 731-02

0'Hearon, Sr. Marie Therese, Good Counsel Convent, 52 N. Broadway, White Plains, NY, 10603

O'Reilly, Michael C., Senior Instructor, Saudi Arabian Airlines, CC 452, P. 0. Box 167, Jeddah, SAUDI ARABIA

Ochoa, A., Department of Education, $650 \mathrm{~W}$ State St-Jordan Bldg., Boise, ID, 83720

Odense Universitetsbibliotek, Campusvej 55, Ok-5230, Odense, Denmark

Ohio State University, Serials Division - Library, 1858 Neil Avenue, Columbus, $\mathrm{OH}, 43210$

OK lahoma Baptist University, Director, Language Laboratory, Shawnee, OK, 74801

Ok lahoma State University, Edmon Low Library, Serials Section, Stillwater, OK, 74078

01 sen, Solveig, P. O. Box 13633 N. T. Station, Denton, TX, 76203

Ort, B., Department of Education, P. 0. Box 30008, Lansing, MI, 48909

0tto, Sue, Computer Assisted Instruction, University of lowa, Language Media, 125 Schaeffer Hall, Iowa City, IA, 52242

P/H Electronics, Inc., Mr. E. R. Hazlett, President and General Manager, $413 \mathrm{E}$. Helena Street, Dayton, $\mathrm{OH}, 45404$

Paramskas, Danute M., Dept. of Language \& Literature, University of Guelph, Guelph, Ontario, CANADA NI6 $2 W 1$

Parr, Susan, Language Laboratory, Juniata College, Huntingdon, PA, 16652

Patron, Gloria A., Language Laboratory, University of New Orleans, Lake Front, New Orleans, LA, 70122

Pennsylvania State Library, Serial Records Section, Box 1601, Room 46, Harrisburg, PA, 17105

Pensacola Junior College, Library, 1000 College Boulevard, Pensacola, FL, 32504

Penta, Richard M., Coord., Foreign Language, Belmont School System, 38 Normandy Road, Lexington, MA, 02173

Petersen, Gerald W., Dept. of Foreign Languages, University of Nevada, Reno, NV, 89507

Peterson, Greg, Nortre Dame Women's College, Shimogamo, Sakyo-ku, Kyoto, JAPAN 606

Petherbridge, D. L., University of Lethbridge, Lethbridge, Al., CANADA TIK $3 M 4$

Pflanz, Barbara, Dept. of German, University of Redlands, Redlands, CA, 92373

Pickell, Ruth, Language Lab, Chaminade Julienne High School, 505 South Ludlow Street, Dayton, $\mathrm{OH}, 45402$

Pierce, James A., Department of English, Del Mar College - East, Corpus Christi, TX, 78404

Pineyro, R., Department of Education, 721 Capitol Mall, Sacramento, CA, 95814

Plagerman, Mark, Language Lab, Miami University-Middletown, 4200 Manchester Road, Middletown, $\mathrm{OH}, 45052$

Pleasants, Jeanne Varney, 2815 Treasure Is land East, Memphis, TN, 38115

Pohlmeier, R., Educational Media Division, Inc., P. O. Box 20604, Ok lahoma City, OK, 73156 
Powell, Richard K., Teaching Materials \& Learning Ctr., Andrews University, Berrien Springs, MI, 49104

Pratt, William, A/V Media Specialist, College of Virgin Islands, St. Thomas, VI, 00801

Pritchard, C., National Sales Manager, Special Markets, SONY OF CANADA LTD., 411 Gordon Baker Road, Willowdale, Ontario CANAOA M2H 256

Procopio, Joseph, Language Lab. Coord inator, The Wardl aw-Hartridge School, P.0. Box 1882-Muhlenberg Station, Plainfield, NJ, 07060

Professional Resource Center, Attn: Ms. B. Katz, Wayne County Intermediate District, 33400 Van Born Road, Wayne, MI, 48184

Prueau, Albert, Campus Notre-Dame-de-Foy, 5000, rue Saint-Felix, Cap-Rouge, Que., CANADA GOA IKO

Purdue University, PCF - Kardex - Libraries, 19573/Serials, West Laf ayette, IN, 47907

Queens College, Serials Librarian, Flushing, NY, 11367

Ramsay, Patricia L., Instructional Support Center, Eastern Michigan University, 102 Library, Ypsilanti, MI, 48197

Rawlings, Ruth, 101 Kobuke Cho, Chiba Shi, Chiba Ken 281, JAPAN

Raymond, Walter, Atelier de langues, Cegep Ste-Foy, 2410, Chemin Ste-Foy, Ste-Foy, Quebec, CANADA GIV IT3

Reeves, Gaynor E., Language Laboratory, University of Newcastle, Newcastle, NSW, AUSTRALIA 2308

Reimers, Prof. Theresia E., Dept. of Modern Languages, Hollins College, Hollins College, VA, 24020

Renegar, Christopher, Language Laboratory, Occidental College, 1600 Campus Road, Los Angeles, CA, 90041

Rey, Arsenio, University of Alaska, Foreign Language Dept., 3211 Providence Avenue, Anchorage, AK, 99504

Rich, P.J., Box 1300, Deha, Qatar, SÁUDI ARABIA

Richardson, Charles, Language Laboratory, Ohio University, Ellis Hall, Athens, $\mathrm{OH}, 45701$

Richardson, Martha R., Asst. Director, Harvard University, Boylston Hall, G-3, Cambridge, MA, 02138

Richmond, Edmun B., Modern Language Dept., Georgia Institute of Technology, At lanta, GA, 30332

Riekehof, Lottie L., Dept. of Sign Communication, Gallaudet College, Kendall Green, Washington, DC, 20002

Rivers, Wilga M., Prof., Dept. of Romance Languages, Harvard University, 207 Boylston Hall, Cambridge, MA, 02138

Roberts, John E., Intensive English Program, P.0. Box 248005, University of Miami, Coral Gables, FL, 33124

Rodewald, Janet D., Language Laboratory, Central Michigan University, Mt. Pleasant, MI, 48858

Royalty, James E., Language Media Center, University of Maryland, College Park, MD, 20742

Rubin, Joan, NCBR, P.0. Box 143, Pinole, CA, 94564

Russell, Martha A., 49 Gay Street, Norwood, MA, 02062

SEAMCO Reg. Language Center, Librarian of Information Center, 30 Orange Grove Road, SINGAPORE 1025

Saga University, Honjyo-Machi, Saga City, JAPAN $840 \mathrm{MZ}$

Sampon, Victor, Language Laboratory, Wayland Academy, Beaver Dam, WI, 53916

San Diego State University, Language Laboratory, San Diego, CA, 92182

San Diego State University, Love Library-Serials, San Diego, CA, 92182

Sanborn, Frederic R. G. 7480 DeMar Road, Cincinnati, OH, 45243

Sanchez-Berroa, Roger, Director, Language Laboratory, Hesleyan University, Middletown, CT, 06457 
Sapporo Ika Daigaku, Toshokan S-1 W-17, Sapporo, JAPAN 060 (KIN)

Sawyer, Isabel, Language, Linguistics, \& Literature, Simon Fraser University, Burnaby, BC, CANADA V5A IS6

Saylor, Leslie G., Language Laboratory, University of Wisconsin/Milwaukee, P.0. Box 413, Milwaukee, WI, 53201

Schaedel, Gene E., Kay Elemetrics Corporation, 12 Maple Ave., Pinebrook, NJ, 07058

Schladweiler, K., Language Arts, Education Division, Kneip Building, Pierre, SD, 57501

Schrader, Ernst, 15 East 26th St., Rm. 1908A, New York, NY, 10010

Schultz, David, Media Services, Lehman College, Bedford Park Blvd., West, Bronx, NY, 10468

Science \& Technology Info. Center, NTNU, P.0. Box 4 Nankang, Taipei (115), TIAWAN ROC

Scinicariello, Sharon, Language Lab, Muskingum College, New Concord, $\mathrm{OH}$, 43762

Sclafani, Charles, Language Laboratory, Westchester Community College, Valhalla, NY, 10595

Seidel, J., Language Laboratory, University of Victoria, P.0. Box 1700, Victoria, BC, CANADA VBW 2 Y2

Seinan Gakuin University, Language Lab, 6-2-92 Nishijin Nishi-Ku, Fukuoka, JAPAN 814

Seng, Mark W., University of Texas, EDB 406 C\&I, Austin, TX, 78712

Sheehan, Joseph H., Language \& Culture Center, Department of English, University of Houston, Houston, TX, 77004

Shogo Miura, 72-121, Jike, Sayo-cho, Hiroshima, JAPAN 724

Simon Fraser University, Library - Acquisition Division, Burnaby, BC, CANADA V5A IS6

Simone, Mary, Language Lab, University of Mass/Boston, Harbor Campus, Boston, MA, 02125

Sivertsen, Tor, Tandberg of America, Inc., Labriola Court, P.0. Box 58, Armonk, NY, 10504

Smith, William Flint, Department of Modern Languages, Purdue University, LaFayette, IN, 47907

Snyder, Ada P., 1231 Juniper Ct., Fort Collins, C0, 80521

Sokalski, Alex, Language Labs, Dept. of French and Spanish, University of Saskatchewan, Saskatoon, CANADA S7N OWO

Somner, Ursula, Director, Instructional Media, Fairleigh Dickinson University, 285 Madison Avenue, Madison, NJ, 07940

Soumokil, P., Bilingual Education, Hathaway Building, Cheyene, WY, 82002

Southern Illinois University, Morris Library, Periodical Department, Carbondale, IL, 62901

Spinks, 0.R., Humanities B-16, State University of, New York at Albany, Albany, NY, 12222

St. Michael's College, Director, Intern. Student Program, Attn: Norman Lacharite, Winooski, VT, 05404

Stadt und Universität sbibliothek, Bockenheimer Landstrasse 134, 6000 Frankfurt, WEST GERMANY

Staley, H., Education Department, P.0. Box 771, Jackson, MS, 39205

Stanford University, Library - Serials Department, Stanford, CA, 94305

State Univ. of NY at Binghamton, Library - Serial Section, Vestal Parkway East, Binghamton, NY, 13901

State University College, Butler Library, Box P, 1300 Elmwood Avenue, Buffalo, NY, 14222

Stockholms Universitet, Larostudion, UIf Essen, Stockholm, SWEDEN S-106 91

Strei, Gerry, BLE/TESOL, Nova University, 6191 SW 45th Street, Davie, FL, 33314

Stromberg, Wayne, Foreign Language Lab, California State University, College of Arts and Letters, San Diego, CA, 92183

Sugimori, Mikihiko, UU 232 Brittany Pl ace, Lawrence, KS, 66044 
Suzugamine Women's, College Library, Inokuchi Nishiku, Hiroshima, $733 \mathrm{MZ}$ JAPAN

Swinyard, Or. Sharon J., Language Laboratory, St. Joseph's College, Box 7009, Mountain View, CA, 94039

Tanner, Jackie, Language Learning Technology, School of Languages \& Linguistics, George Washington University, Washington, DC, 20057

Tantramar Regional High School, French Department, Box 1060, Sackville, NB, CANADA EOA BCO

Teachers College Library, Serials Dept., Serials Dept, Box 307, 525 W. 120 St., New York, NY, 10027

Texas Tech University, Germanic \& Slavic Languages, P.0. Box 4579, Lubbock, TX, 79409

Thomas, J. E., Learning Laboratory, Virginia Polytechnic Institute, Blacksburg, VA, 24061

Tokyo-Denki-Daigaku, Gaikokugo-Keiretsu, Kanda-Nishiki-Cho, 101 Tokyo MZ, JAPAN

Tokyo-Seitoku-Tanki-Daigaku, Library (MZ), 7-13, Jujodai-1-Chome, Kita-Ku, Tokyo, JAPAN

Tool, David N., Humanities - A/V Center, University of Southern California, Los Angeles, CA, 90007

Tracy, Robert K., Director, Language Lab., Ridgewood High School, - Ridgewood, NJ, 07450

Trometer, Ruth, Language Lab, M.I.T., 14N-220, Cambridge, MA, 02139

USAF Academy, Library DFSLBS, Colorado Spring, CO, 80840

Union College, Library, 3800 S. 48th Street, Lincoln, NE, 68506

University Microfilms International, Serials Development, Publisher Relations \& Development, 300 North Zeeb Road, Ann Arbor, MI, 48106

Universidad de Concepcion, Biblioteca Central, Casilla 1807, Concepcion, CHILE

Universität Bern, Institut fur Sprachwissenschaft, Abteilung fur angewandte Linguistik, Hallerstrasse, SWITZERLAND 12

Un iversität sbibliothek, Ze itschriften-FGSZ, Universitätsstrasse 4, Erlangen, WEST GERMANY 8520

Universite de Neuchatel, Chaire de Linguistique Generale, Neuchatel, SWITZERLAND 2000

Universiteitsbibliotheek, 1429 A.324.639, Erasmuslaan 36, Nijmegen, HOLLAND

University of Alberta, Language Laboratory, Attn: C. Dechaine, Edmonton, Alberta, CANADA T6G 2EI

University of California, Director, Language Laboratory, B-40 Dwinelle H Hall, Berkeley, CA, 94720

University of California-San Diego, Language Laboratory C-008, LaJolla, CA, 92093

University of Chicago, Language Lab, 1126 E. 59 Street, Chicago, IL, 60637

University of Colorado, Library, Serials, Boulder, 10,80309

University of Dayton, Periodical Department, Roesch Library - Periodical Dept., 300 College Park Avenue, Dayton, OH, 45469

University of Georgia, Libraries - Periodicals Desk - LT, Athens, GA, 30602

University of Illinois, Language of Learning Laboratory, G-70 FLB, Urbana, IL, 61801

University of Illinois - Urbana, Serials Dept. Library - FAX, Urbana, IL, 61801

University of Kansas Libraries, Periodicals - Serials, Lawrence, KS, 66045

University of Kentucky, Language Laboratory, 312 Classroom Building, Lexington, $\mathrm{KY}, 40506$

University of Louisville, Attn: Dayle Manges, Serials Department Library, Louisville, KY, 40292 
University of Melbourne, Medley Building, West Tower, The Horwood Language Centre, Parkville, Vic., AUSTRALIA 3052

University of Minnesota, Serial Records - Libraries, Minneapolis, MN, 55455

University of Nebraska - Omaha, Gene Eppley Library, Periodicals Department, 60th \& Dodge Streets, Omaha, NE, 68182

University of Nevada, Library - Periodicals Dept., Las Vegas, NV, 89154

University of Nevada, Library - Serials Dept., Reno, NV, 89557

University of queensiand, Main Library, Serials Librarian, St. Lucia, Que., AUSTRALIA 4067

University of S. Africa at Port Elizabeth, Library-Periodicals Dept., Private Bag 6058, Port Elizabeth, SOUTH AFRICA 6000

University of South Dakota, I.D. Weeks Library, Vermillion, SD, 57069

University of Southwestern Louisiana, Dupre Library, 302 East St. Mary Blvd., LaF ayette, LA, 70504

University of St. Andrews, Modern Language Library, Attn: Ms. Konn, Buchanan Building, Union Street, St Andrews, Fife, SCOTLAND KYI6 9PH

University of Stellenbosch, Library, Private Bag 5036, Stellenbosch, SOUTH AFRICA 7600

University of Sydney, Fischer Library, Serials Division 134881, Sydney, NSW, AUSTRALIA 2006

University of Texas, General Libraries, Central Serials Record, Austin, TX, 78712

University of Toledo, Carlson Library, Serials Department, Toledo, OH, 43606

University of Turku, Language Centre, 20500, Turku 50, FINLAND

University of Western Ontario, Periodicals Department, General Library, London, Ontario, CANADA N6A 3K7

University of Wisconsin, Library - Serials Division, Parkside, Wood Road, Kenosha, WI, 53141

University of Wisconsin, Library - Serials Department, 728 State Street, Madison, WI, 53706

University of Zimbabwe, Language Laboratory \#3643, P.0. Box MP 167, Mount Pleasant, Salisbury, ZIMBABWE

Uptis, Graciela, 175 Churchill Lane, Lincoln, IL, 62656

Van Dyke, Jerry, Fleetwood Furniture Co., Zeeland, MI, 49464

Van 01 phen, Herman, Language Laboratory, University of Texas at Austin, Batts Hall 1, Austin, TX, 78712

Vavak, Max, Language Lab, California State University, 1250 Bellflower Bivd., Long Beach, CA, 90840

Vernick, Judy A., Language Laboratory, University of Pittsburgh, G-17 C of $L$, Pittsburgh, $P A, 15260$

Vijayakumar, E. K., Thurber Comprehensive High School, 15 Orillia Park, Red Deer, CANADA T4N 5 A6

Voge, Wilfried, Language Laboratory, University of California, Humanities Hal1, Rm 263, Irvine, CA, 92717

Waguespack, P., Language Education, P. O. Box 44064, Baton Rouge, LA, 70804

Walsh, Terence M., Director of Research, St. Paul's School, Pleasant Street, Concord, NH, 03301

Wa lter, Jim, Language Lab, Sinclair Community College, 444 West 3rd Street, Dayton, $\mathrm{OH}, 45402$

Warkentin, Henry, Foreign Language Department, Shippensburg State College, Shippensburg, PA, 17257

Warriner, H., Bilingual Education, P.0. Box 6Q, Richmond, VA, 23216

Washington English Research Assoc., 5761 Buckingham Parkway, Attn: Edward Hinson, Culver City, CA, 90230 
Watson, John A., Language Laboratory, Virginia Union University, Richmond, VA, 23220

Weible, David M., Audio Information Service, Univ. of Illinois at Chicago, Box 4348, Rm. 203, Grant Hall, Chicago, IL, 60680

We inkauf, Arnold L., Department of Languages, Michigan Technological University, Houghton, MI, 49931

Wesleyan University, Language Laboratory, 116 Fisk Hall, West Station, Middletown, CT, 06457

West Georgia College, School of Arts and Sciences, Dept. of Foreign Languages, Caroliton, GA, 30118

West Texas State University, Cornette Library, Box 748 W. T. Station, Canyon, TX, 79016

Wickert, Gabriele, Language Resource Center, Manhattanville College, Purchase, NY, 10577

Wilde, Karen, 1605 N. Fillmore St., Arlington, VA, 22201

Willecke, F. H., Foreign Language Department, Wagner College, Staten Island, NY, 10301

William, Charles B., Language Laboratory, State University of New York, Oswego, NY, 13126

Willis, Robert M., Communications - Arts \& Sciences, 829 Shimk in Hall, 50 West 4th Street, New York, NY, 10003

Wilson, Kirk, President, Learning Tools, Inc., 686 Massachusetts Ave., Cambridge, MA, 02139

Wilson, Sandra, Marietta Jr. High School, 7th \& Glendale Sts., Marietta, $\mathrm{OH}, 45750$

Wingert, Iris R., Instructional Media Resources, Language Ctr. Academic IV Rm. 219, Univ. of Maryland-Baltimore County, Catonsville, MD, 21228

Wipf, Joseph, 121 East Knox Drive, West LaFayette, IN, 47906

Wollstein, John D., Educational Specialist, Asian, European \& Pacific Languages, 1270 queen Emma St., \#1106, Honolulu, HI, 96813

Wyatt, David H., English for Special Purposes, American Language Academy, 11426 Rockville Pike, Suite 200, Rockville, MD, 20852

Yates, Janis M., Jeffrey Norton Publishers, Inc., On the Green, Guilford, CT, 06437

York University, Central Serials Records, 4700 Keele Street, Downsview, Ont., CANADA M3J 2R2

Young, Dale, Kobe Steel Language Center, Kobe Steel, Ltd., Fuji Bldg 7 Floor, 1-8-2, Marunouchi, Chiyoda-ku, Tokyo, JAPAN 103

Yzenbaard, James 0 ., Director, The Language Lab., University of Michigan, 2015 Modern Language B1dg., Ann Arbor, MI, 48109

Zaetta, R., Department of Instruction, Townsend Building, Dover, DE, 19901

Zimmerman, Mrs. M., Language Laboratory, Amherst College, Amherst, MA, 01002

Zimerman, Sr. Dorothy M., Fourier Laboratory, Mount Mary College, Milwaukee, WI, 53222

GEOGRAPHICAL LIST

ALABAMA

Cox, Shirley 0., Language Laboratory, Auburn University, Auburn, AL, 36830

Gleaves, Glen, Language Laboratory, University of South Alabama, Mobile, $\mathrm{AL}, 36688$

Hirsch, Bernice G., Foreign Language Lab, Samford University, 800

Lakeshore Drive, Birmingham, AL, 35229 
Howard, J., Department of Education, 111 Coliseum Blvd., Montgomery, AL, 36109

ALASKA

Rey, Arsenio, University of Alaska, Foreign Language Dept., 3211

Providence Avenue, Anchorage, AK, 99504

ARIZONA

Arizona State University, Library - Periodicals, Tempe, AZ, 85281

Maynes, J., Department of Education, 1535 West Jefferson, Phoenix, AZ, 85007

ARKANSAS

Cheatham, Rosalie M., Language Laboratory, Arkansas University at Little Rock, 6405 Evergreen Road, Little Rock, AR, 72207

Hudson, G., Department of Education, Little Rock, AR, 72201

CALIFORNIA

Blinco, Priscilla N., 196 Stewart Drive, Tiburon, CA, 94920

Bloomer, Joan, Coordinator, Langauage Lab, University of California Davis, Davis, CA, 95616

Bridgers, William K., Learning Laboratories, Instructional Development, University of California, Santa Barbara, CA, 93106

Brinton, Donna, ESL Media Consultant-UCLA, English IESL, $405 \mathrm{Hilgard}$ Avenue, Los Angeles, CA, 90024

California Polytechnic State Univ., University Library - Periodicals , San Luis Obispo, CA, 93407

Comet, Honore, All-Wor ld Language Institute, Language Laboratory - Suite 500,280 South Beverly Drive, Beverly Hills, CA, 90212

DL IWC AC ademic Library, DLIFLC BIdg. 618, M/F DAKF03-80-M-3315, Presidio, Monterey, CA, 93940

DeMers, Irene, Harvard School, 3700 Coldwater Canyon Road, North Hollywood, CA, 91604

Educational Electronics of Calif., Attn: Bernard Keach, 213 North Cedar Avenue, Inglewood, CA, 90301

Ford, Kathleen, Administrative Assistant, Foreign Language - Instruc. Lab., 362 Royce Hall, UCLA, Los Angeles, CA, 90024

Forth, Joli, 973 Kingston Avenue, Piedmont, CA, 94611

Froehlich, Jurgen, Language Laboratory, Pomona College, Claremont, CA, 91711

Grant, R. M., 2345 Filbert Street, No. 202, San Francisco, CA, 94123

Hall, Bunelle M., Monterey Institute of International Studies, 425 Van Buren Street, Monterey, CA, 93940

Hendriks, Helga M. Leonhardt, Foreign Language Insturctor, 640 San Elijo Street, San Diego, CA, 92106

Hutchinson, Joseph C., P.0. Box 0J, Pacific Grove, CA, 93950

Kessler, Michael, School of Humanities, 1600 Holloway, San Francisco State University, San Francisco, CA, 94132

Kirk, David, 124 Chace Street, Santa Cruz, CA, 95060

Language \& Language Behavior Abs., P.0. Box 22206, San Diego, CA, 92122

Metcalfe, John, Director, Stanford University Language Lab, Stanford, CA, 94305

Pflanz, Barbara, Dept. of German, University of Redlands, Redlands, CA, 92373

Pineyro, R., Department of Education, 721 Capitol Mall, Sacramento, CA, 95814

Renegar, Christopher, Language Laboratory, Occidental College, 1600 Campus Road, Los Angeles, CA, 90041

Rubin, Joan, NCBR, P.0. Box 143, Pinole, CA, 94564

San Diego State University, Language Laboratory, San Diego, CA, 92182 
San Diego State University, Love Library-Serials, San Diego, CA, 92182

Stanford University, Library - Serials Department, Stanford, CA, 94305

Stromberg, Wayne, Foreign Language Lab, California State University, College of Arts and Letters, San Diego, CA, 92183

Swinyard, Or. Sharon J., Language Laboratory, St. Joseph's College, Box 7009, Mountain View, CA, 94039

Tool, David N., Humanities - A/V Center, University of Southern California, Los Angeles, CA, 90007

University of California, Director, Language Laboratory, B-40 Dwinelle $H$ Hall, Berkeley, CA, 94720

University of California-San Diego, Language Laboratory C-008, LaJolla, CA, 92093

Vavak, Max, Language Lab, California State University, 1250 Bellflower Bivd., Long Beach, CA, 90840

Voge, Wilfried, Language Laboratory, University of California, Humanities Hal1, Rm 263, Irvine, CA, 92717

Washington English Research Assoc., 5761 Buckingham Parkway, Attn: Edward Hinson, Culver City, CA, 90230

COLORADO

Butler, Samuel R., 1095 South Downing Street, Denver, C0, 80209

Gilmore, Roger H., Assoc. Prof. of Spanish, Colorado State University/, Foreign Languages, Fort Collins, $\mathrm{CO}, 80523$

Macinko, John, Language Laboratories, University of Colorado, Hellems 156, Box 239, Boulder, CO, 80309

Snyder, Ada P., 1231 Juniper Ct., Fort Collins, C0, 80521

USAF ACademy, Library DFSLBS, Colorado Spring, CO, 80840

University of Colorado, Library, Serials, Boulder, CO, 80309

CONNECTICUT

Capretz, Pierre J., Director, Language Lab., Yale University, 111 Grove Street, New Haven, CT, 06511

Language Instruction Supervisor, Bilingual Education, P.0. Box 2219, Hartford, CT, 06115

Sanchez-Berroa, Roger, Director, Language Laboratory, Wesleyan University, Middletown, CT, 06457

Wesleyan University, Language Laboratory, 116 Fisk Hall, West Station, Middletown, CT, 06457

Yates, Janis M., Jeffrey Norton Publishers, Inc., On the Green, Guilford, CT, 06437

DELAWARE

Zaetta, R., Department of Instruction, Townsend Building, Dover, DE, 19901

DISTRICT OF COLUMBIA

Association for Educational Comm., Attention: John J. Faber, 1201 Sixteenth St., N.W., Washington, DC, 20036

Bernier, Marc, 2164 Florida Avenue, NW, Washington, DC, 20008

Caldwel1, William A., The American University, LFS Asbury, Rm. 326, 4400 Massachusetts Ave., NW, Washington, DC, 20016

Columbus Memorial Library, Serials Pub Sec, OAS, 17th \& Constitution, Washington, DC, 20006

ERIC Exchange, Center for Applied Linguistics, 3520 Prospect Street, N.W., Washington, DC, 20007

Howard University, Language Lab/903301, Room 361 Locke Hall, 2441 6th Street, N.W., Washington, DC, 20059

Howard University Library, Acquisitions Dept. Serials, Room \#105, Washington, DC, 20059

Jo int National Comm. for Languages, J. David Edwards, Director, 11 DuPont Circle, N.W., Suite 210, Washington, DC, 20036 
Laroche, J., Languages, D.C. Public Schools, 415 12th Street, N.W., Washington, DC, 20004

Library of Congress, Order Division, Attn: Section C, Washington, DC, 20540

National Institute of Education, Educational Research Library, Serials Section, Washington, DC, 20208

O'Brien, Paula Sullivan, Dept. of Linguistics/ESL, Trinity College, 124 Michigan Ave., NE, Washington, DC, 20017

Riekehof, Lottie L., Dept. of Sign Comunication, Gallaudet College, Kendall Green, Washington, DC, 20002

Tanner, Jackie, Language Learning Technology, School of Languages \& Linguistics, George Washington University, Washington, DC, 20057

FLORIDA

Arnold, Robert L., Instructional Resources, University of Central Florida, Box 25,000, Orlando, FL, 32816

Florida State University, R. M. Strozier Library, Serials Department, Tallahassee, FL, 32306

Frechette, Ernest A., Foreign Language Education, Florida State University, Tallahassee, $\mathrm{FL}, 32306$

Goldsworth, Thomas, P.0. Box 537, Odessa, FL, 33556

Johansen, Kjell M., Language Laboratory, FL Dept., North Texas State University, Denton, TX, 76203

LHTEC, 905 Lindsey Hopk ins Library, 1410 NE 2nd Avenue, Miami, FL, 33132

Lindsey Hopkins, Library 82, L.H.T.E.C., 750 N.W. 20th Street, Miami, FL, 33136

Pensacola Junior College, Library, 1000 College Boulevard, Pensacola, FL, 32504

Roberts, John E., Intensive English Program, P.0. Box 248005, University of Miami, Coral Gables, FL, 33124

Strei, Gerry, BLE/TESOL, Nova University, 6191 SW 45th Street, Davie, FL, 33314

GEORGIA

Feagin, C., Department of Education, Education Annex-Trinity, Atlanta, $\mathrm{GA}, 30303$

Herrera, Lazaro M., Language Laboratory, The Westminster Schools, 1424 W. Paces Ferry Road, N.W., At lanta, GA, 30327

Mercer University, Language Lab, Attn: Chairman, Dept. of Modern Foreign Languages, Macon, GA, 31207

Morris Brown College, Plant Operations-Receiving, 643 Mart in Luther King, Jr., Dr., Atlanta, GA, 30314

Richmond, Edmun B., Modern Language Dept., Georgia Institute of Technology, At lanta, GA, 30332

University of Georgia, Libraries - Periodicals Desk - LT, Athens, GA, 30602

West Georgia College, School of Arts and Sciences, Dept. of Foreign Languages, Caroliton, GA, 30118

GUAM

Barnes, J., Department of Education, Agana, GU, 96910

HAWAII

Chang, Gerald K. J., Foreign Lanaguage Laboratory, Moore Hall 256, 1890 East-West Road, Honolulu, HI, 96822

Kamehameha Schools, Attn: Bill Gemmer, Kapalma Heights, Honolulu, HI, 96817

Wollstein, John D., Educational Specialist, Asian, European \& Pacific Languages, 1270 Queen Emma St., \#1106, Honolulu, HI, 96813 
IDAKO

Hughett, Harvey, Dept. of Foreign Languages \& Lit., College of Letters \& Science, University of Idaho, Moscow, ID, 83843

Ochoa, A., Department of Education, $650 \mathrm{~W}$ State St-Jordan Bldg., Boise, ID, 83720

\section{ILLINOIS}

Bailey, Leslie F., Language Laboratories, 48B Kresge, 1859 Sheridan Rd., Northwestern University, Evanston, IL, 60201

Be 11, Anne B., 4903 44th Avenue, Moline, IL, 61265

Gillespie, Netta, Language Laboratory, University of 111 inois, 6-89 Foreign Languages, Urbana, IL, 61801

Governors State University, University Library, Serials Dept. - Kardex, Park Forest S., IL, 60466

Griffith, P., Education, 100 North First Street, Springfield, IL, 62777

Heather, Anthony J., Language Resource Center, Loyola University, Chicago, IL, 60626

Heilenman, Laura K., Dept. of French \& Italian, Northwestern University, Evanston, IL, 60201

Mostek, Karlene, 1625 W. Lunt, Chicago, IL, 60626

Southern Illinois University, Morris Library, Periodical Department, Carbondale, IL, 62901

University of Chicago, Language Lab, 1126 E. 59 Street, Chicago, IL, 60637

University of 111 inois, Language of Learning Laboratory, G-70 FLB, Urbana, IL, 61801

University of Illinois - Urbana, Serials Dept. Library - FAX, Urbana, IL, 61801

Uptis, Graciela, 175 Churchill Lane, Lincoln, IL, 62656

Weible, David M., Audio Information Service, Univ. of Illinois at Chicago, Box 4348, Rm. 203, Grant Hall, Chicago, IL, 60680

INDIANA

Bartz, W., Public Instruction, State House 229, Indianapolis, IN, 46204

McCune, Jerry, Language Laboratory, Indiana University, Ballantine Hall 120, Bloomington, IN, 47401

Purdue University, PCF - Kardex - Libraries, 19573/Serials, West Lafayette, IN, 47907

Smith, William Flint, Department of Modern Languages, Purdue University, LaFayette, IN, 47907

Wipf, Joseph, 121 East Knox Drive, West LaFayette, IN, 47906

IOWA

Iowa State University, English/IEOP, 203 Ross Hall, Ames, IA, 50011

Irving, Evelyn Uhrhan, 2508 Glen Elm Drive, N. E., Cedar Rapids, IA, 52402

Otto, Sue, Computer Assisted Instruction, University of Iowa, Language Media, 125 Schaeffer Hall, Iowa City, IA, 52242

KANSAS

Garinger, Ermal E., Language Laboratories, University of Kansas, 4069 Wescoe Hall, Lawrence, KS, 66045

Nicholson, C., Department of Education, 120 East 10th Street, Topeka, KS, 66612

Sugimori, Mikihiko, UU 232 Brittany Place, Lawrence, KS, 66044

University of Kansas Libraries, Periodicals - Serials, Lawrence, KS, 66045

\section{KENTUCKY}

Altman, Howard B., Dept. of Modern Languages, University of Louisville, Louisville, KY, 40292

Brown, C. P., Foreign Language Department, Ivan Wilson Building 251, Western Kentucky University, Bowling Green, KY, 42101

Hershel, Kenneth, 3222 Murray Hill Pike, Louisville, KY 40222 
Koester, A., Department of Education, Frankfort, KY, 40601

Lally, Jr., D. V., Arts \& Sciences Learning Lab, University of Louisville, Louisville, KY, 40292

Murray, David R., AV Services, University of Kentucky, 111 Scott St. B]dg., Lexington, KY, 40506

University of Kentucky, Language Laboratory, $312 \mathrm{Classroom} \mathrm{Building,}$ Lexington, KY, 40506

University of Louisville, Attn: Dayle Manges, Serials Department Library, Louisville, KY, 40292

LOUISIANA

Abed, A. Z., Foreign Language Laboratory, Louisiana State University, Baton Rouge, LA, 70803

Beveridge, Martha J., 8127 Birch Street, New Or leans, LA, 70118

Knox, H. Todd, Language Laboratory, University of Southwestern Louisiana, Box 43331, Lafayette, LA, 70506

Mcleese State University, Frazar Memorial Library, Serials Department, Lake Charles, LA, 70609

Meneghini, Marc, 7214 St. Charles Avenue, New Orleans, LA, 70118

Novak, Sigrid Scholtz, Dept. of Modern Languages, McNeese State University, Lake Charles, LA, 70609

Nuffer, J. Stanley, Learning Laboratory, Portland State University, P.0. Box 751, Port land, OR, 97207

Patron, Gloria A., Language Laboratory, University of New Orleans, Lake Front, New Orleans, LA, 70122

University of Southwestern Louisiana, Dupre Library, 302 East St. Mary B1vd., LaFayette, LA, 70504

Waguespack, P., Language Education, P. 0. Box 44064, Baton Rouge, LA, 70804

MAINE

Abraham, Ruth, Director, Language Lab., Bowdoin College, Sills Hall, Brunswick, ME, 04011

Creamer, Jr., John F., Director, Language Lab, Hudson High School, Brigham Street, Mudson, ME, 01749

MARYLAND

Au lestia, Victor H., Language Media Center, University of Maryland, 5401 Wilkens Avenue, Baltimore, MD, 21228

Beusch, A., Department of Education, P.0. Box 8717, BWI Airport, Bait imore, MD, 21240

Bowie State College, Thurgood Marshall Library, Pullen Library Periodicals, Bowie, MD, 20715

Clark, D. J., Gilman Hall, Johns Hopkins University, Baltimore, MD, 21218

DuVerile, Claude, Modern Languages and Liguistics, UMBC, Catonsvilie, MD, 21228

Hughes, Ann M., Language Lab, Loyola College in Maryland, 4501 N. Charles St., Baltimore, MD, 21210

Magi 11, Robert, Language Lab, Dept. of Modern Language, Towson State University, Baltimore, MD, 21204

Modl in, Columbus M., 2817 W. North Ave., Baltimore, MD, 21216

Royalty, James E., Language Media Center, University of Maryland, College Park, MD, 20742

Wingert, Iris R., Instructional Media Resources, Language Ctr. Academic IV Rm. 219, Univ. of Maryland-Baltimore County, Catonsvilie, MD, 21228

Wyatt, David H., English for Special Purposes, American Language Academy, 11426 Rockville Pike, Suite 200, Rockville, MD, 20852 


\section{MASSACHUSETTS}

Boudreau, Jacqueline, Language Laboratory, Wheaton College, Norton, MA, 02766

Carlson, Florence, Director, Language Lab., Wellesley College, Wellesley, $M A, 02181$

Dente, Edmund, Conte Language Lab, Tufts University, Cabot Center, Rm. 202, Medford, MA, 02155

Department of Education, Director, Bilingual Education, Boston, MA, 0211

Earle, Betty B., Language Lab, Newton South High School, Needham, MA, 02192

Enos, Jacquel ine, Foreign Language Dept., Bridgewater State College, Bridgewater, MA, 02324

Fritsch, Hans J., P.0. Box 61, Whately, MA, 01093

Heinle \& Heinle Publishers, Inc., Attn: Charles Heinle, 286 Congress Street, Boston, MA, 02210

Nicholson, Cynthia, Supervisor, Language Lab., Boston College, Lyons 313, Chestnut Hill, MA, 02167

Northeastern University, Learning Resources Center, 406 Dodge, 360 Huntington Ave., Boston, MA, 02115

Penta, Richard M., Coord., Foreign Language, Belmont School System, 38 Normandy Road, Lexington, MA, 02173

Richardson, Martha R., Asst. Director, Harvard University, Boylston Hall, G-3, Cambridge, MA, 02138

Rivers, Wilga M., Prof., Dept. Of Romance Languages, Harvard University, 207 Boylston Hall, Cambridge, MA, 02138

Russell, Martha A., 49 Gay Street, Norwood, MA, 02062

Simone, Mary, Language Lab, University of Mass/Boston, Harbor Campus, Boston, MA, 02125

Trometer, Ruth, Language Lab, M.I.T., 14N-220, Cambridge, MA, 02139

Wilson, Kirk, President, Learning Tools, Inc., 686 Massachusetts Ave., Cambridge, MA, 02139

Zimmerman, Mrs. M., Language Laboratory, Amherst College, Amherst, MA, 01002

\section{MICHIGAN}

Calvin College Library, 3207 Burton, S. E., Grand Rapids, MI, 49506

Mason, Richard G., 403 N. Frankl in Street, P. 0. Box 26, Houghton, MI, 49931

Ort, B., Department of Education, P. 0. Box 30008, Lansing, MI, 48909

Powell, Richard K., Teaching Materials \& Learning Ctr., Andrews University, Berrien Springs, MI, 49104

Professional Resource Center, Attn: Ms. B. Katz, Wayne County Intermediate District, 33400 Van Born Road, Wayne, MI, 48184

Ramsay, Patricia L., Instructional Support Center, Eastern Michigan University, 102 Library, Ypsilanti, MI, 48197

Rodewald, Janet D., Language Laboratory, Central Michigan University, Mt . Pleasant, MI, 48858

University Microfilms International, Serials Development, Publisher Relations \& Development, 300 North Zeeb Road, Ann Arbor, MI, 48106

Van Dyke, Jerry, Fleetwood Furniture Co., Zeeland, MI, 49464

Weinkauf, Arnold L., Department of Languages, Michigan Technological University, Houghton, MI, 49931

Yzenbaard, James 0., Director, The Language Lab., University of Michigan, 2015 Modern Language Bldg., Ann Arbor, MI, 48109

\section{MINNESOTA}

Carleton College, Library, Northf ield, MN, 55057

Jebe, S., Department of Education, 649 Capitol Square, 550 Cedar Street, St. Paul, MN, 55101 
Johnson, Tom, Audio-Visual Marketing, TELEX COMMUNICATIONS, INC., 9600 Aldrich Avenue South, Minneapol is, MN, 55420

Lange, Dale L., University of Minnesota, 130B Peik Hall, 159 Pillsbury Dr., S.E., Minneapolis, MN, 55455

Messner, C. A., Modern Language, Carleton College, Northfield, MN, 55057

Moorhead State University, Library Serials Department, Moorhead, MN, 56560

University of Minnesota, Serial Records - Libraries, Minneapolis, MN, 55455

MISSISSIPP I

Staley, H., Education Department, P.0. Box 771, Jackson, MS, 39205

MISSOURI

Berndt, Robert J., Dept. of Foreign Language, Southwest Missouri State University, Springfield, MO, 65802

de Johnson, Teresa H., Department of Modern Languages, St. Louis University, St. Louis, MO, 63103

Francis, Larry P., Intensive English Program, University of Missouri, 228 Gentry, Columbia, M0, 65211

King, R., Education Department, P. 0. Box 480, Jefferson City, M0, 65102

Kump, Richard J., 1822 Ricardo Drive, Cape Girardeau, MO, 63701

MONTANA

Jackson, D., Second Languages, Public Instruction, Helena, MT, 59601

NEBRASKA

Coffey, Thomas F., Classics \& Modern Languages Dept., Creighton University, Omaha, NE, 68178

Conner, Maurice W., Central States Conference, University of Nebraska, P. 0 . Box 688, Omaha, NE, 68101

Dalstrom, E. Kay, University of Nebraska, Foreign Languages, Omaha, NE, 68112

Gi lde, Hans M., 01dfather 1111, University of Nebraska, Lincoln, NE, 68588

Mellgren, Millies, 3009 F. Street, Lincoln, NE 68510

Nielson, M., Education Department, 301 Centennial Mall South, Lincoln, NE, 68509

Union College, Library, 3800 S. 48th Street, Lincoln, NE, 68506

University of Nebraska - Omaha, Gene Eppley Library, Periodicals Department, 60th \& Dodge Streets, Omaha, NE, 68182

NEVADA

Abrams, W., Education Department, Capital Complex, Carson City, NY, 89710

Petersen, Gerald W., Dept. Of Foreign Languages, University of Nevada, Reno, NV, 89507

University of Nevada, Library - Periodicals Dept., Las Vegas, NV, 89154

University of Nevada, Library - Serials Dept., Reno, NV, 89557

NEW HAMPSHIRE

Bowen, Louise H., 201 Bartlett Hall, Dartmouth College, Hanover, NH, 03755

Fournier, R., Bilingual Education, 64 N. Main Street, 3rd Floor, Concord, $\mathrm{NH}, 03301$

Goulet, Sister Noella, Notre Dame College, 2321 Elm Street, Manchester, NH, 03104

Walsh, Terence M., Director of Research, St. Paul's School, Pleasant Street, Concord, NH, 03301

NEW JERSEY

Frost, Arthur F., Director, Language Lab., Princeton University, 304 E. Pyne Building, Princeton, NJ, 08540 
Procopio, Joseph, Language Lab. Coordinator, The Wardlaw-Hartridge School, P.0. Box 1882-Muhlenberg Station, Plainfield, NJ, 07060

Schaedel, Gene E., Kay Elemetrics Corporation, 12 Maple Ave., Pinebrook, NJ, 07058

Sormer, Ursula, Director, Instructional Media, Fairleigh Dickinson University, 285 Madison Avenue, Madison, NJ, 07940

Tracy, Robert K., Director, Language Lab., Ridgewood High School, Ridgewood, NJ, 07450

NEW MEXICO

Kurtz, Don, Language Learning Center, Department of Foreign Languages, Box 3L, Las Cruces, NM, 88003

NEW YORK

ADFL Bulletin, Editor, 62 Fifth Avenue, New York, NY, 10011

Bator, Joseph W., Language Development, Pan American World Airways John F. Kennedy Airport, Jamaica, NY, 11430

Bronx Community College-CUNY, Dept. Library \& Learning Resources, University Ave. \& W 181 St., Bronx, NY, 10453

Criminale, Leonard R., Language Laboratory, Elmira College, Elmira, NY, 14901

Dammer, P., Foreign Language Education, Education Department, Albany, NY, 12234

de la Portilla, Marta, Spanish Department, Manhattanville College, Purchase Street, Purchase, NY, 10577

DeTorre, Emilio E., Queens College, City University of New York (CUNY), Flushing, NY, 11367

Hamilton College, Serials Dept., Clinton, NY, 13323

Kruse, Jr., E. L., 733 Kirkwood Drive, Grand Island, NY, 14072

Levinson, Bernice, Language Laboratory, Brooklyn College-CUNY, Modern Languages \& Literatures, Brooklyn, NY, 11210

Marino, Anna, Language Laboratory, Hunter College, 695 Park Avenue, Box 375, New York, NY, 10021

Martin, Leslie, Columbia-Green Community College, P.0. Box 1000, Hudson, NY, 12534

McCarty, Jean, Language Laboratory, 106 Calkins Ha11, Hofstra University, Hempstead, NY, 11550

Modern Language Association, Advertising Manager, 62 Fifth Avenue, New York, NY, 10011

Nassau Community College, Library - Periodicals Unit, Stewart Avenue, Garden City, NY, 11530

Nelson, R., College Department, John Wiley \& Sons, 605 3rd Avenue, New York, NY, 10016

Norton, Jeffrey, Jeffrey Norton Publishers, Inc., Audio-Forum, 145 E. 49th Street, New York, NY, 10017

0 'Hearon, Sr. Marie Therese, Good Counsel Convent, 52 N. Broadway, White Plains, NY, 10603

Queens College, Serials Librarian, Flushing, NY, 11367

Schrader, Ernst, 15 East 26th St., Rm. 1908A, New York, NY, 10010

Schultz, David, Media Services, Lehman College, Bedford Park Blvd., West, Bronx, NY, 10468

Sclafani, Charles, Language Laboratory, Hestchester Cormunity College, Valhalla, NY, 10595

Sivertsen, Tor, Tandberg of America, Inc., Labriola Court, P.0. Box 58, Armonk, NY, 10504

Spinks, 0.R., Humanities B-16, State University of, New York at Albany, Albany, NY, 12222

State Univ. of NY at Binghamton, Library - Serial Section, Vestal Parkway East, Binghanton, NY, 13901 
State University College, Butler Library, Box P, 1300 Elmwood Avenue, Buffalo, NY, 14222

Teachers College Library, Serials Dept., Serials Dept, Box 307, 525 W. 120 St., New York, NY, 10027

Wickert, Gabriele, Language Resource Center, Manhattanville College, Purchase, NY, 10577

Willecke, F. H., Foreign Language Department, Wagner College, Staten Is land, NY, 10301

William, Charles B., Language Laboratory, State University of New York, Oswego, NY, 13126

Willis, Robert M., Communications - Arts \& Sciences, 829 Shimkin Hall, 50 West 4th Street, New York, NY, 10003

NORTH CAROL INA

Alyta, Kenneth J., 5316 Chedworth Drive, Charlotte, NC, 28210

Appalachian State University, Serials Department, Bulk Library, Boone, NC, 28608

D.H. Hill Library, Periodicals Service Center, Box 5007-NC State, Raleigh, NC, 27650

Inf ante, J., Languages, Public Instruction Department, Raleigh, NC, 2761

NORTH DAKOTA

Lacher, S., Languages, Public Instruction, Capitol Building, Bismarck, ND, 58505

OHIO

Aronovsky, Mrs., Bishop Hartley High School, 1285 Zettler Road, Columbus, $\mathrm{OH}, 43227$

Baker, Reid E., Foreign Language Supervisor, Education Department, 65 South Front Street, Columbus, $\mathrm{OH}, 43215$

Benseler, David P., MODERN LANGUAGE JOURNAL, 314 Cunz Ha11, Ohio State University, Columbus, $\mathrm{OH}, 43210$

Bleznick, Donald W., Editor, HISPANIA, Romance Languages, University of Cincinnati, Cincinnati, $\mathrm{OH}, 45221$

Boggins, Judy, Foreign Language Lab, Raymond Walters College, 9555 Plainfield Road, Cincinnati, $\mathrm{OH}, 45221$

Boyden, Patrick C., Self-Instruction Center, 271 Library, Kent State University, Kent, $\mathrm{OH}, 44242$

Christman, Sandra, Foreign Language Department, Chillicothe High School, 381 Yoctangee Pkwy., Cillicothe, $\mathrm{OH}, 45601$

Cleveland State University, Library Order Unit, Serials, 1860 E. 22nd Street, Cleveland, $\mathrm{OH}, 44115$

Coltharp, Jerry, Audio Visual Service, 340 Gaskill Hall, Miami University, Oxford, $\mathrm{OH}, 45056$

Courtright Memorial Library, Otterbein College SAN 313-7937, Westerville, $\mathrm{OH}, 43081$

Dill, David, Foreign Language Dept., Lancaster High School, 1312 Granvilie Pike, Lancaster, $\mathrm{OH}, 43130$

Fox, Jean, Language Department, Columbus School for Girls, 56 South Columbia Avenue, Columbus, $\mathrm{OH}, 43209$

Frank, Stefania, University Hall, Bowling Green State University, Bowling Green, $\mathrm{OH}, 43402$

Griffin, Howard, Denison University-A/V Services, Granvill, OH, 43023

Hanby, Mr., Principal, New Lexington Jr. High School, Route 2, New Lexington, $\mathrm{OH}, 43764$

Harnagle, James, Language Department, University of Steubenville, College Heights, Steubenville, OH, 43952

Herdler, Mrs., Romance Language Dept., Walnut Hills High School, 3250 Victory Pkwy., Cincinnati, OH, 45207 
Hockenson, Mrs., Language Lab, Cincinnati Country Day School, 6905 Given Road, Cincinnati, $\mathrm{OH}, 45243$

Jackson, Gordon, 257 Cliffview Drive, Gahanna, OH, 43230

Larson, H. M., Languages \& Literatures, Cedarville College, Cedarville, $\mathrm{OH}, 45314$

Lianez, David, Language Lab, Van Wert High School, Crawford \& Jefferson, Van Wert, $\mathrm{OH}, 45891$

Loud, Mary, Foreign Languages, Youngstown State University, Youngstown, $\mathrm{OH}, 44555$

Marietta College, Modern Language Dept., Marietta, $\mathrm{OH}, 45760$

Miller, J. B., Wittenberg University, Springfield, $\mathrm{OH}, 45501$

Miller, William I., Modern Languages, University of Akron, 31001 in Hall, Akron, $\mathrm{OH}, 44325$

Ohio State University, Serials Division - Library, 1858 Neil Avenue, Columbus, $\mathrm{OH}, 43210$

P/H Electronics, Inc., Mr. E. R. Hazlett, President and General Manager, $413 \mathrm{E}$. Helena Street, Dayton, $\mathrm{OH}, 45404$

Pickell, Ruth, Language Lab, Chaminade Julienne High School, 505 South Ludiow Street, Dayton, OH, 45402

Plagerman, Mark, Language Lab, Miami University-Middletown, 4200 Manchester Road, Middletown, $\mathrm{OH}, 45052$

Richardson, Charles, Language Laboratory, Ohio University, Ellis Hall, Athens, $\mathrm{OH}, 45701$

Sanborn, Frederic R. G., 7480 DeMar Road, Cincinnati, OH, 45243

Scinicariello, Sharon, Language Lab, Muskingum College, New Concord, $\mathrm{OH}$, 43762

University of Dayton, Periodical Department, Roesch Library - Periodical Dept., 300 College Park Avenue, Dayton, OH, 45469

University of Toledo, Carlson Library, Serials Department, Toledo, $\mathrm{OH}$, 43606

Walter, Jim, Language Lab, Sinclair Community College, 444 West 3rd Street, Dayton, $\mathrm{OH}, 45402$

Wilson, Sandra, Marietta Jr. High School, 7th \& Glendale Sts., Marietta, $\mathrm{OH}, 45750$

\section{OKLAHOMA}

ELS Educational Media, Attn: Shelby Hardage, P. 0. Box 20604, Ok lahoma City, OK, 73156

Educational Media Division, Attn: Jim Goodin, P. 0. Box 20604, 2812 Quail Plaza Drive, OKlahoma City, OK, 73156

Ok lahoma Baptist University, Director, Language Laboratory, Shawnee, OK, 74801

Ok lahoma State University, Edmon Low Library, Serials Section, Stillwater, OK, 74078

Pohlmeier, R., Educational Media Division, Inc., P. 0. Box 20604, Oklahoma City, OK, 73156

OREGON

Booth, Trudie, Dept. of French, University of Portland, 5000 N. Willanete Bivd., Portland, OR, 97203

PENNSYLVANIA

Al aghelley, Abdulaziz M., 200 N. Negley Ave., Apt. E-42, Pittsburgh, PA, 15206

Chestnut, D., Education Department, Education Building, Box 911, Harrisburg, PA, 17126

Conwell, Marilyn J., 251 West Dekalb Pike, Apt. 909C, King of Prussia, PA, 19406

Entin, Nathaniel A., Gratz College, 10th Street \& Tabor Road, Philadelphia, PÁ, 19141 
Fisher, Ronald D., Language Laboratory, LaSalle College, 20th Street at 01ney Avenue, Philadelphia, PA, 19141

Francis H. Green Library, West Chester State College, West Chester, PA, 19380

Gennaula, M. G., 5872 Kings School Road, Bethel Park, PA, 15102

Kalenchic, Mary Beth, Learning Center, Lehigh University, 401 Coppee Hall 33, Bethlehem, PÁ, 18015

Kipphorn, Jr., Richard A., Language Laboratory, Saint Joseph's University, Philadelphia, PA, 19131

Kogut, Daniel E., Wickersham Hall, Millersville State College, Millersville, $P A, 17551$

LaVallee-Hms., Marthe, 1326 Spruce Street, No. 3001, Philadelphia, PA, 19107

Lawrason, Robin E., Media Learning Center, Temple University, Philadelphia, PA, 19122

Parr, Susan, Language Laboratory, Juniata College, Huntingdon, PA, 16652

Pennsylvania State Library, Serial Records Section, Box 1601, Room 46, Harrisburg, PA, 17105

Vernick, Judy A. , Language Laboratory, University of Pittsburgh, G-17 C of $L$, Pittsburgh, PA, 15260

Warkentin, Henry, Foreign Language Department, Shippensburg State College, Shippensburg, $P A, 17257$

PUERTO RICO

Inter-American University, Library, San German, PR, 00753

RHODE ISLAND

Brown University, Language Laboratory Library, Box E, Providence, RI, 02912

SOUTH CAROLINA

Arnold, E. P., 201 Strode Tower, Clemson University, Clemson, SC, 29631

Cherry, Charles Maurice, Modern Language Laboratory, Furman University, Greenville, SC, 29613

Galloway, V., Foreign Language Consultant, Education Department, 801 Rut ledge, Columbia, SC, 29201

SOUTH DAKOTA

Schladweiler, K., Language Arts, Education Division, Kneip Building, Pierre, SD, 57501

University of South Dakota, I.D. Weeks Library, Vermillion, SD, 57069

\section{TENNESSEE}

Allen, Andrew, Romance Languages Dept., University of Tennessee, Knoxville, TN, 37996

Dinkelacker, Horst, Language Center, Southwestern at Memphis, $2000 \mathrm{~N}$. Parkway, Memphis, TN, 38112

Education Language Dept., 1067 Buford Ellington, Kennedy Campus, Memphis, TN, 38111

Harwood, Sharon, Dept. of Foreign Language, Memphis State University, Memphis, TN, 38152

Morrison., Robert R., Southern Missionary College, P.0. Box 475, Collegedale, TN, 37315

Pleasants, Jeanne Varney, 2815 Treasure Is land East, Mermphis, TN, 38115

TEXAS

Al len, Ernest, Language Laboratory, Texas Christian University, 1 Allen Farm Road, Fort Worth, TX, 761 i6

Ambard, Francois, Colorado Apts., K201, Lake Austin Blvd., Austin, TX, 78703 
Barakat, Ghias, P.0. Box 8333, Aust in, TX, 78712

Barria, Jose G., 4521 Leeds Avenue, EI Paso, TX, 79903

Cate, Robert L., Modern Language Laboratory, University of Texas at El Paso, El Paso, TX, 79968

Gionet, Arthur J., Dept. of Foreign Languages \& Lit., North Texas State University, Denton, TX, 76203

Mckay, Jym M., Dept. of Speech Communication, University of Texas, Aust in, TX, 78712

Menin, J. L., Director, Language Laboratory, Rice University, P.0. Box 1892, Houston, TX, 77251

North Texas State University, Library, P. O. Box 5188 NT Station, Denton, TX, 76203

01 sen, Solveig, P. 0. Box 13633 N. T. Station, Denton, TX, 76203

Pierce, James A., Department of English, Del Mar College - East, Corpus Christi, TX, 78404

Seng, Mark W., University of Texas, EDB 406 C\&I, Austin, TX, 78712

Sheehan, Joseph H., Language \& Culture Center, Department of English, University of Houston, Houston, TX, 77004

Texas Tech University, Germanic \& Slavic Languages, P.0. Box 4579, Lubbock, TX, 79409

University of Texas, General Libraries, Central Serials Record, Austin, $T X, 78712$

Van 01phen, Herman, Language Laboratory, University of Texas at Austin, Batts Hall 1, Austin, TX, 78712

West Texas State University, Cornette Library, Box 748 W. T. Station, Canyon, TX, 79016

UTAH

Brigham Young University, Dr. Jerry W. Larson, Humanities Research Center, 130B-34, Provo, UT, 84602

Brigham Young University, Harold Lee Library, Serials Section, Provo, UT, 84602

Burggraaf, Samuel E., W-162 Stadium, Brigham Young University, Provo, UT, 84602

Howe, E., Foreign Language Education, Education Department, 250 East 500 South, Salt Lake City, UT, 84111

Luckau, Paul F., Germanic Languages, Brigham Young University, 270 MSRD, Provo, UT, 84602

VERMONT

Dodge, James W., Secretary/Treas., Northeast Conf., Box 623, Middlebury, VT, 05753

Gore, Peter B., P.0. Box 22, Wilmington, VT, 05363

St. Michael's College, Director, Intern. Student Program, Attn: Norman Lacharite, Winooski, VT, 05404

VIRGIN ISLANDS

Language Instruction Supervisor, Education Department, P.0. Box 630, St. Thomas, VI, 00801

Pratt, William, A/V Media Specialist, College of Virgin Islands, St. Thomas, VI, 00801

VIRGINIA

Hi 11, Ann R., Language Laboratory, Randolph-Macon Woman's College, 2400 Rivermont Avenue, Lynchburg, VA, 24503

Instructional Materials Center, 201 East Nine Mile Road, Highland Spring, VA, 23075

Little, William A., GERMAN QUARTERLY, Germanic and Slavic Languages, University of Virginia, Charlottesville, VA, 22901 
Reimers, Prof. Theresia E., Dept . of Modern Languages, Hollins College, Hollins College, VA, 24020

Thomas, J. E., Learning Laboratory, Virginia Polytechnic Institute, Blacksburg, $V A, 24061$

Warriner, H., Bilingual Education, P.0. Box 6Q, Richmond, VA, 23216

Watson, John A., Language Laboratory, Virginia Union University, Richmond, $V A, 23220$

Wilde, Karen, 1605 N. Fillmore St., Arlington, VA, 22201

\section{WASHINGTON}

Al len, Nina A., Language Learning Center, $\mathrm{DH}-40$, University of Washington, Seattle, WA, 98195

Augerot, James E., Language Learning Center, University of Washington, Seattle, WA, 98195

Bocaz-Moraga, Sergio, Dept. of Modern Languages, Eastern Washington University, 3144 Patterson Hall, Cheney, WA, 99004

Crosbie, K., Bilingual Instruction, Public Instruction, 01d Capitol Building, Olympia, WA, 98504

Elmendorf, William E., Language Laboratory, Western Washington State College, Bellingham, WA, 98225

Johnson, Barbara, Foreign Lanaguage Department, Washington State University, Pullman, WA, 99164

\section{WISCONSIN}

Bosworth, Lewis A., New Student Services, 905 Univ. Ave., Suite 1, University of Wisconsin, Madison, WI, 53715

Grittner, F., Foreign Language Education, Public Instruction, 126 Langdon, Madison, WI, 53702

Hoff, Roma, University of Wisconsin-Eau Claire, 335 Bartlett Court, Eau Claire, WI, 54701

Labs for Recorded Instruction, Attn: Dr. R. G. Gilgen, 279 Van Hise Hall, 1220 Linden Drive, Univ. of Wisc., Madison, WI, 53706

Latinovich, Gina, Language Lab, Marquette University, 526 N. Fourteenth St., Milwaukee, WI, 53233

Sampon, Victor, Language Laboratory, Wayland Acadeny, Beaver Dam, WI, 53916

Saylor, Leslie G., Language Laboratory, University of Wisconsin/Milwaukee, P.O. Box 413, Mi 1waukee, WI, 53201

University of Wisconsin, Library - Serials Division, Parkside, Wood Road, Kenosha, WI, 53141

University of Wisconsin, Library - Serials Department, 728 State Street, Madison, WI, 53706

Zimmerman, Sr. Dorothy M., Fourier Laboratory, Mount Mary College, Milwaukee, WI, 53222

WYOMING

Soumokil, P., Bilingual Education, Hathaway Building, Cheyene, WY, 82002

ARGENTINA

de Buhler, Angela Vidal, Pampa 1940, 1428 Buenos Aires, ARGENTINA

\section{AUSTRALIA}

Auchmuty Library, Serials Dept., University of Newcastle, Newcastle, NSW, AUSTRALIA 2308

Clark, C. A., Australian National University, Instructional Resources Unit, Chifley Library, Box 4, Canberra, A C T, AUSTRALIA 2600

Dowling, J. K., Language Laboratory, University of Queensland, St. Lucia, Bris, AUSTRALIA 4067 
Jordan, Terence C., Language Centre, Griffith University, Nathan, Queens., AUSTRALIA 4111

McAndrew, Alex, Univ. of Sydney-Language Study Ctr., Sydney, NSW, AUSTRALIA 2006

Monash University, Periodicals Department, Clayton, Vic, AUSTRALIA 3168

National Library of Australia, Preliminary Processing, (NS 110/72), ACT 2600, Canberra, AUSTRALIA

Reeves, Gaynor E., Language Laboratory, University of Newcastle, Newcast le, NSW, AUSTRALIA 2308

University of Melbourne, Medley Building, West Tower, The Horwood Language Centre, Parkville, Vic., AUSTRALIA 3052

University of Queensland, Main Library, Serials Librarian, St. Lucia, Que., AUSTRALIA 4067

University of Sydney, Fischer Library, Serials Division 134881, Sydney, NSW, AUSTRALIA 2006

\section{BELGIUM}

Applied Ling. Institute of Belgium, Attn: Willy Martin, Blijde Inkomststraat 21, Leuven, BELGIUM 3000

BOLIVIA

Jabalquinto, Sergio Rios, Casilia 165, Tarija, BoLIVIA

CANADA

Aikens, H. F., Part-time Studies \& Extension, Dalhousie University, Halifax, NS, CANADA B3H 335

Aubert, Jean-Philippe, McGill University, 3438, McTavish Street, Montreal, CANADA H3A $1 \times 9$

Aucoin, G. E., 34 Langley Avenue, Dartmouth, Nova Scotia, CANADA B2W 2 Y6

Balcaen, Hubert L., Language Laboratories, University of Manitoba, Winnipeg, CANADA R3T 2 M8

Brandon University, John E. Robbins Library, Brandon Man., CANADA R7A6A9

Canadian Forces Base St. - Jean, Base Commander, Unit B - Rm. F-267, Richelain, Quebec JOJ IRO CANADA

Caron, Paul, Dept. of English, CEGEP Riviere du Loup, 80 Frontenac, Riviere du Loup, Quebec 65R IV8 CANADA

Carter, Thomas P., Learning Resource Services, Dalhousie University, Killam Library, Halifax, NS, CANADA B3H $4 \mathrm{H} 8$

Ciceran, A. J., Language Laboratory, Brock University - Decew Campus, St. Catharines, Ontario, CANADA L2S 3AI

College of Cape Breton, Library - Sydney Campus, Box 5300, Sydney, NS, CANADA BIP 6L2

Concordia University, Libraries-Serials-Acquisitions, P.0. Box 2650, Montreal, Que., CANADA H3G 2P7

Couroux, Gerard, College Marie Victorin, 7000 rue Marie Victorin, Montreal, Que., CANADA 462

Ham, C., Language Laboratories, Queen's University, Kingston, Ont., CANADA K7L 3N6

Hammerly, Hector, Simon Fraser University, Literature \& Linguistics, Burnaby, BC, CANADA V5A IS6

Hennig, C. W., Language Laboratory, University of Waterloo, Waterloo, Ont., CANADA N2L 3G1

Hewson, J., Linguistics, Memorial University of Newfoundland, St. John's NFD, CÁNADA

Holmes, Glyn, French, University of Western Ontario, London, CANADA N6A $3 \mathrm{~K} 7$ 
Kenner, R., H-399 Concordia University, Language Laboratory Division-AV, 1455 de Maisonneuve Blvd., Montreal, Que., CANADA H3G IM8

Kozoriz, G., Canadian Forces Lang. Schl-ottowa, Nat'1. Defense Hdq. (NOHQ), Ot towa, Ontario, CANADA KIA OK2

Lemyze, J. C., Language Laboratory, McGill University, Peterson Hall, 3460 McTavish Street, Montreal, Que., CANADA H3A ixg

Majhanovich, Suzanne E., Faculty of Education, University of Windsor, Windsor, Ont., CANADA N9B 3P4

Marxheimer, Edward, Romance Languages, University of Alberta, Edmonton, Alberta, CANADA T6G 2E6

McGill University Libraries, Serials Department, 3459 McTavish Street, Montreal, CANADA H3A IYi

Ministry of Culture \& Recreation, Resource Centre, 77 Bloor Street West, Toronto, Ontar., CANADA M7A 2R9

National Library of Canada, Order Section, 395 Wellington Street, Ottawa, Ontario, CANADA KIA ON4

Paramskas, Danute M., Dept. of Language \& Literature, University of Guelph, Guelph, Ontario, CANADA N16 2WI

Petherbridge, D. L., University of Lethbridge, Lethbridge, Al., CANADA TIK $3 M 4$

Pritchard, C., National Sales Manager, Special Markets, SONY OF CANADA LTD., 411 Gordon Baker Road, Willowdale, Ontario CANADA M2H 2S6

Prueau, Albert, Campus Notre-Dame-de-Foy, 5000, rue Saint-Felix, Cap-Rouge, Que., CANADA GOA IKO

Raymond, Walter, Atelier de langues, Cegep Ste-Foy, 2410, Chemin Ste-Foy, Ste-Foy, Quebec, CANADA GIV $1 T 3$

Sawyer, Isabel, Language, Linguistics, \& Literature, Simon Fraser University, Burnaby, BC, CANADA V5A 156

Seide1, J., Language Laboratory, University of Victoria, P.0. Box 1700, Victoria, BC, CANADA VBW 2 Y2

Simon Fraser University, Library - Acquisition Division, Burnaby, BC, CANADA V5A 156

Sokalski, Alex, Language Labs, Dept. of French and Spanish, University of Saskatchewan, Saskatoon, CANADA STN OWO

Tantramar Regional High School, French Department, Box 1060, Sackville, NB, CANADA EOA $8 C 0$

University of Alberta, Language Laboratory, Attn: C. Dechaine, Edmonton, Alberta, CANADA T6G 2EI

University of Western Ontario, Periodicals Department, General Library, London, Ontario, CANADA N6A 3K7

Vijayakumar, E. K., Thurber Comprehensive High School, 15 Orillia Park, Red Deer, CANADA T4N 5A6

York University, Central Serials Records, 4700 Keele Street, Downsview, Ont., CANADA M3J 2R2

CHILE

Baltra, Liliana, (CPEIP), Casilla 16162, Santiago, CHILE 9

Universidad de Concepcion, Biblioteca Central, Casilla 1807, Concepcion, CHILE

DENMARK

Odense Universitetsbibliotek, Campusvej 55, Dk-5230, Odense, Denmark ECUADOR

Lopez, Jose A., Licenciado, ESPOL, Apartado 5863, Guayaqui 1, ECUADOR 303733 
AV Language Journal, Attn: A. P. Dyson, University of Oxford, Lang. Ctr., 41 Wellington Square, Oxford, ENGLAND OXi 2JF

British Library, Lending Division, Accessions Department-Lending Div., Boston Spa, Wetherby, Yorkshire, ENGLAND LS237BQ

Center for Information on, Language Teaching, Deputy Librarian, 20 Carlton House Terrace, London S.W., ENGLAND IY 5AP

\section{FINLAND}

Helsing in Yliopisto, Kielikeskus, Fabianinkatu 26, SF-00100, Helsinki 10, FINLAND

Korkeakoulun, Joensuun, Kirjasto, PL 111, 80101, Joensuu 10, FINLAND University of Turku, Language Centre, 20500, Turku 50, FINLAND

\section{FRANCE}

Institue de Linguistique Appliquee, Laboratoires de Langues, Universite de Strasbourg, 22, rue Descartes, Strasbourg-Cedx, FRANCE 67084

HOLLAND

Universiteitsbibliotheek, 1429 A.324.639, Erasmuslaan 36, Nijmegen, HOLLAND

HUNGARY

Konyvtar, Egyetemi, POB 483, Budapest 1372, HUNGARY

INDIA

Annamalai, E., I/C Library, Central Inst. of Indian Languages, Manasagangotri, Mysore-570 006, INDIA

ISRAEL

David Yellin Teacher's Seminary, Beth-Hakerem, P.0. Box 3578, Jerusalem, ISRAEL

Jewish National \& Univ. Lib., Attn: Periodicals Dept., P.0. Box 503, Jerusalem, ISRAEL 91004

\section{JAPAN}

Achiba, Machiko, 8-34 Komuinshukusha, 11-72 Sakashita, Myodaiji-cho, Okazaki, Aichi, JAPAN 444

Amano, Kazuo, 16--6 Shiomigaoka-Cho, Chiba-Shi 280, JAPAN

Daigaku, Gifu Jyoshi Tanki, 2693 Fukumitsu, Nagara, Gifu 502 MZ, JAPAN

Daigaku, Kyushu Sangyo, (LL Room), 327, Matugadai-2-chome, Higashi-ku, Fukuoka City, JAPAN 813 (MZ)

Fukuoka University, LL Room, Nanakuma, Fukuoka 814 MZ, JAPAN

Hanze 1, Louis F., Nanzan University, is Yamazato-cho, Showa-ku, Nagoya, JAPAN 466

Hiroshima Daigaku, Honbu Toshokan-K, Higashisendamachi Nakaku, Hiroshima MZ, JAPAN 730

Hiroshima Shudo Daigaku Library, Otsuka Numata-Cho, Hiroshima (Kin), JAPAN 731-31

Honda, Ritsuri, Assumption College, 2-16 0te-cho, Takatsuki-shi, Osaka, JAPAN 569 
Hyogo-Kyoiku Daigaku, Toshokan, Yashiro-Cho Yashiro, Kato-Gun Hyogo, JAPAN 67.3-14

Ibaraki Christ-Kyo, Tanki-Daigaku Library, 6-11-1 Omika-Cho Hitachi, Ibaraki 319-12, JAPAN (KIN)

Ikeura, Sadahiko, Fukuoka University of Education, 279 Akama, Maunakata-gun, Fukuoka, JAPAN

Ishikawa Kosen, Kitachujo, Tsubata, Kahoku-Gun, Ishikawa, JAPAN 929-03 MZ

Kagawa University, Library, Saiwai-Cho, Takamatsu-Shi, JAPAN 760 (KIN)

Kansai University Library, P.O. Box 50, Suita, Osaka, JAPAN 564 MZ

Kenagawa University, Library, Rokkakubashi, Kanagawa-Ku, Yokohama (MZ), JAPAN 221

Kenji Kitao, Dept. of English, Doshisha University, Kyoto, JAPAN 602

Kohmoto, Sutesaburo, 4-16-17 Meguro, Meguro-ku, Tokyo, JAPAN 153

Kure Koygo Koto, Senmon Gakko Library, Aga-Minami-2-2-11, Kure, JAPAN 737 MZ

Kuroda, Takashi, Language Laboratory Assoc. of Japan, Eng. Lit. Dept. Otsuma Womens Col., 12, 3-bancho, Chiyoda-ku, Tokyo, JAPAN 102

Kyoto Sangyo Daigaku, Toshokan, (Kyoyo-Eigo-Ken), Kita-Ku Kyoto, JAPAN $603 \mathrm{MZ}$

Lance, John, Japanese American Conversation Inst, Intl. Education Center, 21 Yotsuya, 1-chome, Shinjuku-ku, JAPAN 160

Maze, Louis M., English Language Program Director, Procter \& Gamble Sunhome Mfg. Co., 321 Yawata-machi, Takasaki-shi, JAPAN 370

Nagoya Gakuin Daigaku, Gaikokugo Center, Kamishinano-Cho, Seto, Aichiken 48012MZ, JAPAN

Nihon Eigo-Kentei-Kyokai, 1 Yaraicho, Shinjuku-ku, Tokyo, JAPAN 162 (SSD)

O'Donohue, Barbara, Communications, Hiroshima Bunkyo Womens College, Kabe Cho-Asa Kita Ku, Hiroshima, JAPAN 731-02

Peterson, Greg, Nortre Dame Women's College, Shimogamo, Sakyo-ku, Kyoto, JAPAN 606

Rawlings, Ruth, 101 Kobuke Cho, Chiba Shi, Chiba Ken 281, JAPAN

Saga University, Honjyo-Machi, Saga City, JAPAN $840 \mathrm{MZ}$

Sapporo Ika Daigaku, Toshokan S-1 W-17, Sapporo, JAPAN 060 (KIN)

Seinan Gakuin University, Language Lab, 6-2-92 Nishijin Nishi-Ku, Fukuoka, JAPAN 814

Shogo Miura, 72-121, Jike, Sayo-cho, Hiroshima, JAPAN 724

Suzugamine Women's, College Library, Inokuchi Nishiku, Hiroshima, 733 MZ JAPAN

Tokyo-Denki-Daigaku, Gaikokugo-Keiretsu, Kanda-Nishiki-Cho, 101 Tokyo MZ, JAPAN

Tokyo-Se itoku-Tanki-Daigaku, Library (MZ), 7-13, Jujodai-1-Chome, Kita-Ku, Tokyo, JAPAN

Young, Dale, Kobe Steel Language Center, Kobe Steel, Ltd., Fuji Bldg 7 Fioor, 1-8-2, Marunouchi, Chiyoda-ku, Tokyo, JAPAN 103

\title{
NEW ZEALAND
}

Norrish, N., Language Laboratory, University of Victoria, Private Bag, Wellington, NEW ZEALAND

\author{
NORWAY
}

Moseby, Erik, Tandberg A/S, P. O. Box 53, 2007-KJELLER, NORWAY

\section{SAUDI ARABIA}

0'Reilly, Michael C., Senior Instructor, Saudi Arabian Airlines, CC 452,

P. 0. Box 167, Jeddah, SALDI ARABIA

Rich, P.J., Box 1300, Deha, Qatar, SAUDI ARABIA 
University of St. Andrews, Modern Language Library, Attn: Ms. Konn, Buchanan Building, Union Street, St Andrews, Fife, SCOTLAND KYí 9PH SINGAPORE

National University of Singapore, Central Library, Serials Department, Kent Ridge, SINGAPORE

SEAMCO Reg. Language Center, Librarian of Information Center, 30 Orange Grove Road, SINGAPORE 1025

\section{SOUTH AFRICA}

University of S. Africa at Port Elizabeth, Library-Periodicals Dept., Private Bag 6058, Port El izabeth, SOUTH AFRICA 6000

University of Stellenbosch, Library, Private Bag 5036, Stellenbosch, SOUTH AFRICA 7600

\section{SWEDEN}

Davies, Noman F., Professor, University of Linkoping, Linköping, SWEDEN S-581 83

Stockholms Universitet, Larostudion, Ulf Essen, Stockholm, SWEDEN S-106 91

\section{SWITZERLAND}

Bennet, T.J.A., Language Laboratories, Hochschule St. Gallen, Dufourstrasse 50, St. Gallen, SWI TZERLAND 9014

Universität Bern, Institut für Sprachwissenschaft, Abteilung für angewandte Linguistik, Hallerstrasse, SWI TZERLAND 12

Universite de Neuchatel, Chaire de Linguistique Qenerale, Neuchatel, SWITZERLAND 2000

\section{TIAWAN}

Science \& Technology Info. Center, NTNU, P.0. Box 4 Nankang, Taipei (115), TIAWAN ROC

\section{USSR}

Krupatkin, Yakov Borisovich, u.1. Odesskaja 1, kv. 19, Sevastopo 1, USSR 335000

\section{WEST GERMANY}

Buchhandlung Lehmkuhl, Leopoldstrasse 45, 8000 Muchen 40, WEST GERMANY Free University of Berlin, Foreign Language Laboratories, Habelschwerdter Al lee 45, Ihnenstrasse 24, 1 Berl in 33, WEST GERMANY

Freudenstein, Reinhold, Foreign Language Research Info. Ctr., Lahnberge, D-3550, Marburg, WEST GERMANY

Stadt und Un iversitätsbibliothek, Bockenheimer Landstrasse 134, 6000 Frankfurt, WEST GERMANY

Universitätsbibliothek, Zeitschriften-FGSZ, Universitätsstrasse 4, Erlangen, WEST GERMANY 8520

\section{ZIMBABWE}

University of Zimbabwe, Language Laboratory \#3643, P.0. Box MP 167, Mount Pleasant, Salisbury, ZIMBABWE 